\title{
Solitary waves in the Nonlinear Dirac Equation
}

Jesús Cuevas-Maraver, Nabile Boussaïd, Andrew Comech, Ruomeng Lan, Panayotis G. Kevrekidis, and Avadh Saxena

\begin{abstract}
In the present work, we consider the existence, stability, and dynamics of solitary waves in the nonlinear Dirac equation. We start by introducing the Soler model of self-interacting spinors, and discuss its localized waveforms in one, two, and three spatial dimensions and the equations they satisfy. We present the associated explicit solutions in one dimension and numerically obtain their analogues in higher dimensions. The stability is subsequently discussed from a theoretical perspective and then complemented with numerical computations. Finally, the dynamics of the solutions is explored and compared to its non-relativistic analogue, which is the nonlinear Schrödinger equation.
\end{abstract}

Jesús Cuevas-Maraver

Grupo de Física No Lineal, Universidad de Sevilla, Departamento de Física Aplicada I, Escuela Politécnica Superior. C/ Virgen de África, 7, 41011-Sevilla, Spain,

Instituto de Matemáticas de la Universidad de Sevilla (IMUS). Edificio Celestino Mutis. Avda. Reina Mercedes s/n, 41012-Sevilla, Spain e-mail: jcuevas@us.es

Nabile Boussaïd

Université de Franche-Comté, 25030 Besançon CEDEX, France

Andrew Comech

St. Petersburg State University, St. Petersburg 199178, Russia

Department of Mathematics, Texas A\&M University, College Station, TX 77843-3368, USA

IITP, Moscow 127994, Russia

Roumeng Lan

Department of Mathematics, Texas A\&M University, College Station, TX 77843-3368, USA

Panayotis G. Kevrekidis

Department of Mathematics and Statistics, University of Massachusetts, Amherst, MA 010034515, USA

Avadh Saxena

Center for Nonlinear Studies and Theoretical Division, Los Alamos National Laboratory, Los Alamos, New Mexico 87545, USA 
Key words: Solitons, solitary waves, vortices, nonlinear Dirac equation, stability, Soler model.

\section{Introduction}

In the last three decades, there has been an enormous interest in the study of waves in nonlinear dispersive media. Arguably, two of the most paradigmatic equations that describe such waves are the nonlinear Schrödinger equation (NLS) and the sine-Gordon equation. The first among these equations covers a broad range of settings including atomic physics [121, 122], nonlinear optics [92,94], condensed matter physics, and mathematical physics [2,147]. The sine-Gordon equation also covers settings in condensed matter physics and mathematical physics apart from high-energy physics models [29,47]. A principal focus of the relevant properties of these equations has been the study of the existence, stability, and dynamics of solitary waves (i.e. spatially localized waves supported by the nonlinearity and dispersion), both in lower-dimensional settings (such as one-dimensional solitons and multi-solitons) and in higher dimensional settings (vortices, vortex rings, and related structures) [50,92].

By comparison, far less attention has been paid to the nonlinear Dirac equation (NLD), despite its presence for almost 90 years in the realm of high energy physics. The nonlinear Dirac equation with scalar-type self-interaction was initially introduced by Ivanenko in 1938 [84]. Following the ideas of Finkelstein [70], Heisenberg in 1957 [81] used this NLD model in an attempt to formulate a unified theory of elementary particles. In 1958, a completely integrable one-dimensional model known as the Massive Thirring Model (MTM) [149], based on vector-type self-interaction of spinor field, was introduced. This model possesses solitary wave solutions. $\mathrm{Cu}-$ riously, fundamental solutions of the MTM can be transformed into solitons of the sine-Gordon equation by means of a bosonization process [35]. In 1970, Soler reintroduced Ivanenko's model with scalar-type self-interaction in the context of extended nucleons [143] and also provided the numerical analysis of solitary wave solutions. The one-dimensional version of the Soler model, known as the Gross-Neveu model [75], was introduced in 1974 as a toy model of quark confinement in quantum chromodynamics, and explicit solitary wave solutions in the corresponding massive model were found by Lee et al. in 1975 [97]. We can not complete this quick review of NLD models in high-energy physics without mentioning the recent work of [112] (see also [106]), where a variant of the NLD is applied to the study of neutrino oscillations. Related systems are the Dirac-Maxwell system [21, 38, 57, 76, 155], the Einstein-Dirac system [131, 146], and Einstein-Dirac-Maxwell system [132]. In quantum chemistry, the Dirac-Hartree-Fock model $[60,61,98]$ takes into account the fermionic properties of electrons (describing the exchange interaction, which is a fundamental effect of purely quantum nature) and is used for accurate computation of the electronic energy $[125,153]$; this model has also started to receive mathematical attention [60-62]. 
Recent years have seen a gradual increase of interest in the study of nearrelativistic settings, arguably, for three principal reasons. In recent years, the study of nonlinear Dirac type models has received a renewed thrust for a variety of reasons. Both one-dimensional $[25,27,37,119]$ and two-dimensional variants of the model have been examined from the perspective of solitary wave solutions and their stability $[15,25,118]$. Furthermore, the computational examination of solitary wave solutions and their dynamics has provided numerous insights on the NLD [41, 44, 140, 157] also on its variant in the presence of external fields [111]. Thirdly, the NLD is emerging as the model of relevance in variety of settings including the dynamics of Bose-Einstein condensates in honeycomb lattices $[78,79]$, as well as in atomically thin 2D Dirac materials $[68,156]$. Relevant examples include, but are not limited to graphene, silicene, germanene, borophene, and transition metal dichalcogenides [103]. One of the most intriguing applications of the model has arisen in recent years in so-called photonic graphene [1,3], i.e., the examination of light propagation in honeycomb photorefractive lattices. This direction has led to the investigation of conical diffraction [115] and the exploration of nonlinear phenomena [1,3]. It is worth highlighting, however, that the nonlinearity in this context breaks the Lorentz symmetry (that is, such models are not invariant under Lorentz transformations; for the explicit form of the Lorentz transformations of the spinor fields see e.g. [18, 148]).

Another recent application involving the interplay of Dirac and Schrödinger operators is that of spin-orbit coupled Bose-Einstein condensates [48]. Their experimental realization $[96,100,124]$ and interplay with nonlinearity (due to interatomic interactions) has led to the exploration of a diverse host of nonlinear states including bright, dark and gap solitons [4, 5, 69, 89, 159], self-trapped states [108], vortices [127, 128, 158], Skyrmions [90], as well as Dirac monopoles [39]. The complex interplay of these different effects may even stabilize vortex solitons against collapse in free space, under attractive interactions [135].

From a mathematical perspective, Dirac models are described by systems (rather than by scalar equations) that correspond to the Hamiltonian functionals unbounded from below. This unboundedness makes all the aspects of the analysis of these models (well-posedness, existence of localized solutions, stability, numerical simulations) much more challenging. This has fueled an increasing interest in the nonlinear Dirac equation and more general models of self-interacting spinor fields, with many results on the existence of solitary waves $[33,59,109]$ and well-posedness in $(3+1) \mathrm{D}[56,101]$ and in $(1+1) \mathrm{D}[31,83,102,116,138]^{1}$. The stability of solitary wave solutions of the nonlinear Dirac equation was approached via numerical simulations [7-9, 27,34,111,126,157] and via heuristic arguments [19,20,41, 107, 145], but it is still not settled. Recently, the first stability results in the context of selfinteracting spinor fields started appearing [22,23, 25-27,37, 118].

The NLD can also be viewed as a relativistic generalization (or extension) of the NLS, or, alternatively, the NLS (with additional terms) can be seen as a special case limit of the NLD at the low-energy limit. Nevertheless, it has turned out that the

\footnotetext{
${ }^{1}$ With the notation $(N+1) D$ we want to denote that the system possesses $N+1$ dimensions, with
} $N$ spatial ones plus time 
Dirac equation as a result of its matrix nature and the fact that it is only first order in spatial derivatives (as opposed to second order in the NLS) has proven far more computationally (and theoretically) challenging, on a number of grounds, than its NLS counterpart. This difficulty has hindered the progress in the study of solitary waves, particularly in two-dimensional and three-dimensional settings. However, recent developments are gradually enabling the study of the stability and dynamical properties of solitary waves in two-dimensional and even three-dimensional Soler models; see for a relevant example [46]. Clearly, however, this process requires numerous additional steps that will present several challenges over the coming years.

The aim of this chapter is to give a review of recent results developed by the authors and their collaborators in the last few years, as well as to present a basic framework of the NLD theory, mainly focused on the Soler model and its variants; this is our principal workhorse model. The content of the chapter covers a wide spectrum of results ranging from existence and stability of solitary waves to numerical methods and dynamics of unstable solutions. Apart from this, we also introduce a NLD model with $\mathscr{P} \mathscr{T}$ symmetry and analyze their principal characteristics.

This Chapter is organized as follows: in Section 2 we start with an introduction to the main nonlinear Dirac equation, namely the Soler model, and tractable expressions for the determination of solitary waves and linearizations at solitary waves in one, two, and three spatial dimensions. Section 3 is devoted to the existence properties of solitary waves and numerical methods for their calculation. Stability analysis from a theoretical and numerical point of view is the topic of Sections 4 and 5, respectively. The dynamics of solitary waves is analyzed in Section 6. We finalize the paper with a summary of the considered results and an outlook on future directions on solitary waves in nonlinear Dirac equations.

\section{The Soler model of self-interacting spinors}

In this section we start with the linear Dirac equation and move on to the Soler model as a principal, Lorentz-invariant variant of the model with scalar self-interactions. We give explicit expressions of linearization at solitary waves in one-, two-, and three-dimensional cases.

\subsection{The Dirac equation}

In December 1927, Paul Dirac arrived at the idea of the first-order relativistically invariant equation [54] that describes massive spin-1/2 relativistic fermions in $(3+$ 1) space-time dimensions:

$$
i \hbar \frac{\partial}{\partial t} \psi(t, x)=\left(-i \hbar c \alpha \cdot \nabla+m c^{2} \beta\right) \psi(t, x), \quad \psi(t, x) \in \mathbb{C}^{4}, \quad x \in \mathbb{R}^{3},
$$


with $\psi$ being the spinor-valued wavefunction, $\alpha \cdot \nabla=\sum_{j=1}^{3} \alpha^{j} \frac{\partial}{\partial x^{j}}$, and $m \geq 0$ the mass of the particle. As usual, we choose in what follows the units so that Planck's constant $\hbar$ and the speed of light $c$ are both equal to one. The self-adjoint $4 \times 4$ matrices $\alpha^{j}, 1 \leq j \leq 3$, and $\beta$ satisfy

$$
\left\{\alpha^{j}, \alpha^{k}\right\}=2 \delta_{j k} I_{4}, \quad\left\{\alpha^{j}, \beta\right\}=0, \quad \beta^{2}=I_{4},
$$

with $I_{N}$ being the $N \times N$ identity matrix and $\{A, B\}=A B+B A$ the anticommutator. According to the Dirac-Pauli theorem (see [54, 113, 154], [148, Lemma 2.25], and also [91, Theorem 7] for general version in odd spatial dimensions), different choices of the matrices $\alpha^{j}$ and $\beta$ are equivalent. The most common choice, known as the Dirac-Pauli representation, is

$$
\alpha^{j}=\left(\begin{array}{cc}
0 & \sigma_{j} \\
\sigma_{j} & 0
\end{array}\right), \quad \beta=\left(\begin{array}{cc}
I_{2} & 0 \\
0 & -I_{2}
\end{array}\right)
$$

with the Pauli matrices given by

$$
\sigma_{1}=\left(\begin{array}{ll}
0 & 1 \\
1 & 0
\end{array}\right), \quad \sigma_{2}=\left(\begin{array}{cc}
0 & -i \\
i & 0
\end{array}\right), \quad \sigma_{3}=\left(\begin{array}{cc}
1 & 0 \\
0 & -1
\end{array}\right) .
$$

In the covariant form, the Dirac equation is written as

$$
i \gamma^{\mu} \partial_{\mu} \psi=m \psi
$$

where $\gamma^{\mu} \partial_{\mu}=\sum_{\mu=0}^{3} \gamma^{\mu} \partial_{\mu}, \partial_{0} \equiv \partial_{t}$, with $\gamma^{\mu}$ being the Dirac $\gamma$-matrices

$$
\gamma^{0}=\beta, \quad \gamma^{j}=\beta \alpha^{j}=\left(\begin{array}{cc}
0 & \sigma_{j} \\
-\sigma_{j} & 0
\end{array}\right), \quad j=1,2,3 .
$$

Matrices $\gamma^{\mu}$ fulfill the anticommutation relation $\left\{\gamma^{\mu}, \gamma^{v}\right\}=2 \eta^{\mu v} I_{4}$, with $\eta^{\mu v}$ being the Minkowski tensor [51]. In other words, $\left(\gamma^{0}\right)^{2}=I_{4}$ and $\left(\gamma^{1}\right)^{2}=\left(\gamma^{2}\right)^{2}=\left(\gamma^{3}\right)^{2}=$ $-I_{4}$. There exists another matrix which anticommutes with $\gamma^{0}$ and $\gamma^{j}, 1 \leq j \leq 3$, which plays an important role in the parity transformation. It is the $\gamma^{5}$ matrix, defined by

$$
\gamma^{5}=i \gamma^{0} \gamma^{1} \gamma^{2} \gamma^{3}=\left(\begin{array}{cc}
0 & I_{2} \\
I_{2} & 0
\end{array}\right)
$$

This matrix is self-adjoint and satisfies $\left(\gamma^{5}\right)^{2}=I_{4}$.

One can immediately generalize the ideas of Dirac to an arbitrary spatial dimension $n \geq 1$, writing the Dirac equation

$$
i \partial_{t} \psi=D_{m} \psi \equiv-i \sum_{j=1}^{n} \alpha^{j} \partial_{j} \psi+\beta m \psi, \quad \psi(t, x) \in \mathbb{C}^{N}, \quad x \in \mathbb{R}^{n},
$$

with $\alpha^{j}, 1 \leq j \leq n$, and $\beta$ being selfadjoint matrices satisfying the relations 


$$
\left\{\alpha^{j}, \alpha^{k}\right\}=2 \delta_{j k} I_{N}, \quad\left\{\alpha^{j}, \beta\right\}=0, \quad\left(\alpha^{j}\right)^{2}=\beta^{2}=I_{N} ; \quad 1 \leq j, k \leq n .
$$

The smallest number of spinor components $N$ for the spatial dimension $n \geq 1$ is obtained in the Clifford algebra theory (see e.g. [67, Chapter 1, §5.3]) and is given by

$$
N=2^{\lfloor(n+1) / 2\rfloor} \text {. }
$$

Notice that this relation implies that in the three-dimensional case $(n=3)$, the number of spinor components $N$ must be at least four.

Equation (2.1) is derived from the following Lagrangian density:

$$
\mathscr{L}_{\text {Dirac }}=\bar{\psi}\left(i \gamma^{\mu} \partial_{\mu}-m\right) \psi
$$

where the so-called Dirac conjugate $\bar{\psi}$ is defined by

$$
\bar{\psi} \equiv \psi^{*} \gamma^{0}
$$

with $\psi^{*}$ the Hermitian conjugate of $\psi$.

\subsection{The Soler model}

In 1938, Russian physicist Dmitri Ivanenko proposed a nonlinear model of selfinteracting electrons, introducing the nonlinear term $(\bar{\psi} \psi) \psi$ to the Dirac equation [84]. This self-interaction term is based on the quantity $\bar{\psi} \psi=\psi^{*} \beta \psi$ which transforms as a scalar under Lorentz transformations. In 1970, Spanish physicist Mario Soler re-introduced this model in order to study, from a classical point of view, extended nucleons interacting with their own electromagnetic field [143,144]. Now this equation (or, rather, its version with an arbitrary function of $\bar{\psi} \psi$ ) is known as the Soler model $[33,59,109]$ :

$$
i \partial_{t} \psi=D_{m} \psi-f(\bar{\psi} \psi) \beta \psi, \quad \psi(t, x) \in \mathbb{C}^{N}, \quad x \in \mathbb{R}^{n},
$$

or, in the covariant form,

$$
i \gamma^{\mu} \partial_{\mu} \psi=(m-f(\bar{\psi} \psi)) \psi
$$

where $f \in C(\mathbb{R}), f(0)=0$. Equation (3) admits solitary wave solutions of the form $\psi(t, x)=\phi_{\omega}(x) e^{-i \omega t}$, with $\phi_{\omega}(x)$ exponentially localized in space $[24,33,59,109$, $143,152]$. In addition, the equation is a $\mathbf{U}(1)$-invariant, relativistically invariant hamiltonian system, with the Hamiltonian represented by the density

$$
\mathscr{H}_{\text {Soler }}(\psi)=\psi^{*} D_{m} \psi-F\left(\psi^{*} \beta \psi\right)
$$

with

$$
F(s)=\int_{0}^{s} f(t) d t
$$


the antiderivative of $f$. Because of the $\psi^{*} D_{m} \psi$-term, this Hamiltonian functional is unbounded from below. The Soler model (3) is also characterized by the Lagrangian density

$$
\mathscr{L}_{\text {Soler }}=\bar{\psi}\left(i \gamma^{\mu} \partial_{\mu}-m\right) \psi+F(\bar{\psi} \psi) .
$$

The $\mathbf{U}(1)$-symmetry of the Soler equation leads to the conservation of the value of the charge functional, given by

$$
Q(\psi(t))=\int_{\mathbb{R}^{n}} \psi(t, x)^{*} \psi(t, x) d x
$$

which is conserved in time (one needs to assume that the solution is smooth enough, allowing the integration by parts). If $\psi(t, x)$ is a solution to (3), then both the charge $Q(\psi(t))$ and the energy $E(\psi(t))=\int \mathscr{H}_{\text {Soler }}(\psi(t, x)) d x$ are conserved in time (formally; that is, as long as $\psi$ is sufficiently smooth).

A common choice of the nonlinearity is $f(s)=|s|^{k}, k>0$; this leads to $F(s)=$ $s|s|^{k} /(k+1)$. We note that the absolute value is needed when $k$ is not an integer since the quantity $s=\bar{\psi} \psi$ could be negative. Let us mention that for $k \in(0,1)$, the function $f(s)=|s|^{k}$ is not differentiable at $s=0$, which leads to certain difficulties in the construction of the solitary waves; see [24].

We want to remark that the cubic Soler model

$$
i \partial_{t} \psi=D_{m} \psi-\bar{\psi} \psi \beta \psi
$$

which appeared in [84, 143], differs from (3) with $f(s)=|s|^{k}, k=1$ :

$$
i \partial_{t} \psi=D_{m} \psi-|\bar{\psi} \psi| \beta \psi
$$

Both equations (5) and (6) are relativistically invariant Hamiltonian systems. In particular, both equations are invariant under the time reversal and parity transformation, which are elements of the complete Lorentz group, given respectively by (see e.g. [18])

$$
\psi_{T}(t, x)=i \gamma^{1} \gamma^{3} K \psi(-t, x),
$$

with $K: \mathbb{C}^{4} \rightarrow \mathbb{C}^{4}$ the complex conjugation, and

$$
\psi_{P}(t, x)=\gamma^{0} \psi(t,-x)
$$

At the same time, since $\bar{\psi}_{C} \psi_{C}=-\bar{\psi} \psi$, where the charge conjugation is given by [18]

$$
\psi_{C}(t, x)=-i \gamma^{2} K \psi(t, x),
$$

equation (6) is invariant under the charge conjugation, while equation (5) is not. Let us mention that the choice of unitary factors in all these three transformations is not important. 
We also point out that the stationary waves $\phi_{\omega} e^{-i \omega t}$ constructed in [33] in the three-dimensional case satisfy $\bar{\phi}_{\omega} \phi_{\omega}>0$ for all $x \in \mathbb{R}^{3}$, thus being solutions to both (5) and (6).

\subsection{One-dimensional Soler model}

The Soler model in one spatial dimension, Eq. (3) with $n=1$, is also known as the Gross-Neveu model [75]. According to relation (2), one can take $N=2$, so that the wavefunction is represented by a bi-spinor (i.e. a spinor with only two complex components). We will choose $\alpha^{1}=-\sigma_{2}, \beta=\sigma_{3}$. In this case, the nonlinear Dirac equation (3) can be written as a system of coupled partial differential equations of the form

$$
\begin{aligned}
& i \partial_{t} \psi_{1}=\partial_{x} \psi_{2}+\left(m-f\left(\left|\psi_{1}\right|^{2}-\left|\psi_{2}\right|^{2}\right)\right) \psi_{1}, \\
& i \partial_{t} \psi_{2}=-\partial_{x} \psi_{1}-\left(m-f\left(\left|\psi_{1}\right|^{2}-\left|\psi_{2}\right|^{2}\right)\right) \psi_{2},
\end{aligned}
$$

where $\psi_{1}, \psi_{2} \in \mathbb{C}$ denote the two components of $\psi(t, x) \in \mathbb{C}^{2}$. Notice that in this equation, the spinor components are coupled both in the dispersive term and within the nonlinearity.

The focus of the present chapter is on solitary wave solutions. To this aim, we will search for standing waves of the form

$$
\psi(t, x)=\phi_{\omega}(x) e^{-i \omega t}, \quad \phi_{\omega}(x)=\left[\begin{array}{c}
v(x, \omega) \\
u(x, \omega)
\end{array}\right] \in \mathbb{R}^{2},
$$

with $v(x, \omega)$ and $u(x, \omega)$ satisfying

$$
\begin{aligned}
& \omega v=\partial_{x} u+\left[m-f\left(v^{2}-u^{2}\right)\right] v, \\
& \omega u=-\partial_{x} v-\left[m-f\left(v^{2}-u^{2}\right)\right] u .
\end{aligned}
$$

Once such standing wave solutions are calculated using the methods explained in Subsection 3.2, their linear stability is considered by means of a Bogoliubov-de Gennes (BdG) linearized stability analysis. That is, given a solitary wave solution $\phi_{\omega}(x) e^{-i \omega t}$ with $\phi_{\omega}(x) \in \mathbb{R}^{2}$, we consider its perturbation in the form $\psi(t, x)=$ $\left(\phi_{\omega}(x)+\rho(t, x)\right) e^{-i \omega t}$, with $\rho(t, x) \in \mathbb{C}^{2}$. Then, the linearized equations on $R(t, x)=$ $[\operatorname{Re}(\rho), \operatorname{Im}(\rho)]^{T} \in \mathbb{R}^{4}$ can be written as (see e.g. [27])

$$
\partial_{t} R=\mathscr{A}_{\omega} R
$$

with

$$
\mathscr{A}_{\omega}=\left[\begin{array}{cc}
0 & L_{-}(\omega) \\
-L_{+}(\omega) & 0
\end{array}\right],
$$

where $L_{+}(\omega)$ and $L_{-}(\omega)$ are the following self-adjoint operators: 


$$
\begin{aligned}
& L_{-}(\omega)=\left(\begin{array}{cc}
m-f(\tau)-\omega & \partial_{x} \\
-\partial_{x} & -m+f(\tau)-\omega
\end{array}\right), \\
& L_{+}(\omega)=L_{-}(\omega)-2 f^{\prime}(\tau)\left(\begin{array}{cc}
v^{2} & -v u \\
-v u & u^{2}
\end{array}\right),
\end{aligned}
$$

with $f(\tau)$ and $f^{\prime}(\tau)$ evaluated at $\tau \equiv v^{2}-u^{2}$.

The potential presence of an eigenvalue with non-zero real part in the spectrum of $\mathscr{A}_{\omega}$ suggests the dynamical instability; the corresponding solitary wave is called linearly unstable. If all the eigenvalues are purely imaginary, then the solitary wave is called spectrally (neutrally) stable.

\subsection{Two-dimensional Soler model}

Taking into account the relation given by expression (2), in two spatial dimensions one can again consider two-component spinors. Following [36], a convenient choice for $\alpha$ and $\beta$ matrices is $\alpha^{1}=\sigma_{1}, \alpha^{2}=\sigma_{2}, \beta=\sigma_{3}$. With this in mind, equation (3) is expressed as

$$
\begin{aligned}
& i \partial_{t} \psi_{1}=-\left(i \partial_{x}+\partial_{y}\right) \psi_{2}+\left[m-f\left(\left|\psi_{1}\right|^{2}-\left|\psi_{2}\right|^{2}\right)\right] \psi_{1}, \\
& i \partial_{t} \psi_{2}=-\left(i \partial_{x}-\partial_{y}\right) \psi_{1}-\left[m-f\left(\left|\psi_{1}\right|^{2}-\left|\psi_{2}\right|^{2}\right)\right] \psi_{2} .
\end{aligned}
$$

In order to simplify further analysis, we use the polar coordinates $r=|\mathbf{r}|$ and $\theta$; then equation (11) takes the form

$$
\begin{aligned}
& i \partial_{t} \psi_{1}=-e^{-i \theta}\left(i \partial_{r}+\frac{\partial_{\theta}}{r}\right) \psi_{2}+\left[m-f\left(\left|\psi_{1}\right|^{2}-\left|\psi_{2}\right|^{2}\right)\right] \psi_{1}, \\
& i \partial_{t} \psi_{2}=-e^{i \theta}\left(i \partial_{r}-\frac{\partial_{\theta}}{r}\right) \psi_{1}-\left[m-f\left(\left|\psi_{1}\right|^{2}-\left|\psi_{2}\right|^{2}\right)\right] \psi_{2},
\end{aligned}
$$

with $r \in(0, \infty)$ and $\theta \in[0,2 \pi)$. The form of this equation suggests to search for stationary (standing wave) solutions in the form

$$
\psi(t, \mathbf{r})=\phi_{\omega}(\mathbf{r}) e^{-i \omega t}, \quad \phi_{\omega}(\mathbf{r})=\left[\begin{array}{c}
v(r, \omega) e^{i S \theta} \\
i u(r, \omega) e^{i(S+1) \theta}
\end{array}\right],
$$

with $v(r, \omega)$ and $u(r, \omega)$ real-valued. The value $S \in \mathbb{Z}$ can be cast as the vorticity of the first spinor component. Thus, according to equations (12) and (13), the equations for the stationary solutions read as follows: 


$$
\begin{aligned}
& \omega v=\left(\partial_{r}+\frac{S+1}{r}\right) u+\left[m-f\left(v^{2}-u^{2}\right)\right] v, \\
& \omega u=-\left(\partial_{r}-\frac{S}{r}\right) v-\left[m-f\left(v^{2}-u^{2}\right)\right] u .
\end{aligned}
$$

This set of equations only depends on the radial coordinate $r$. The absence of angular coordinates turns the determination of stationary solutions into a one-dimensional problem, substantially simplifying the numerics.

To examine the spectral stability of a solitary wave, we consider a solution $\psi$ in the form of a perturbed solitary wave:

$$
\psi(t, \mathbf{r})=\left[\begin{array}{c}
\left(v(r, \omega)+\xi_{1}(t, r, \theta)+i \eta_{1}(t, r, \theta)\right) e^{i S \theta} \\
i\left(u(r, \omega)+\xi_{2}(t, r, \theta)+i \eta_{2}(t, r, \theta)\right) e^{i(S+1) \theta}
\end{array}\right] e^{-i \omega t},
$$

with small perturbations $\xi(t, r, \theta)=\left[\xi_{1}, \xi_{2}\right]^{T} \in \mathbb{R}^{2}, \eta(t, r, \theta)=\left[\eta_{1}, \eta_{2}\right]^{T} \in \mathbb{R}^{2}$.

The linearized equation on $R(t, r, \theta)=\left[\xi_{1}, \xi_{2}, \eta_{1}, \eta_{2}\right]^{T} \in \mathbb{R}^{4}$ has the form

$$
\partial_{t} R=\mathscr{A}_{\omega} R
$$

with $\mathscr{A}_{\omega}\left(r, \theta, \partial_{r}, \partial_{\theta}\right)$ a matrix-valued first order differential operator

$$
\mathscr{A}_{\omega}\left(r, \theta, \partial_{r}, \partial_{\theta}\right)=\left[\begin{array}{cc}
-\sigma_{1} \frac{\partial_{\theta}}{r} & L_{-}(\omega) \\
-L_{+}(\omega) & -\sigma_{1} \frac{\partial_{\theta}}{r}
\end{array}\right]
$$

where

$$
\begin{aligned}
& L_{-}(\omega)=\left(\begin{array}{cc}
m-f(\tau)-\omega & \partial_{r}+\frac{S+1}{r} \\
-\left(\partial_{r}-\frac{S}{r}\right) & -m+f(\tau)-\omega
\end{array}\right), \\
& L_{+}(\omega)=L_{-}(\omega)-2 f^{\prime}(\tau)\left(\begin{array}{cc}
v^{2} & -v u \\
-v u & u^{2}
\end{array}\right),
\end{aligned}
$$

with $f(\tau)$ and $f^{\prime}(\tau)$ evaluated at $\tau \equiv v^{2}-u^{2}$.

To find the spectrum of the operator $\mathscr{A}_{\omega}$, we consider it in the space of $\mathbb{C}^{4}$-valued functions. The key observation which facilitates a computation of the spectrum is that the explicit form (16) of $\mathscr{A}_{\omega}$ contains $r, \partial_{r}, \partial_{\theta}$, but not $\theta$. As a consequence, $\mathscr{A}_{\omega}$ is invariant in the spaces which correspond to the Fourier decomposition with respect to $\theta$,

$$
\mathscr{X}_{q}=\left\{\left[a_{1}(r) ; a_{2}(r) ; b_{1}(r) ; b_{2}(r)\right] e^{i q \theta}\right\} \quad q \in \mathbb{Z}
$$

The restriction of $\mathscr{A}_{\omega}$ to each such subspace is given by 


$$
\mathscr{A}_{\omega, q}\left(r, \partial_{r}\right)=\left.\mathscr{A}_{\omega}\right|_{\mathscr{X}_{q}}=\left[\begin{array}{cc}
-\sigma_{1} \frac{i q}{r} & L_{-}(\omega) \\
-L_{+}(\omega) & -\sigma_{1} \frac{i q}{r}
\end{array}\right], \quad q \in \mathbb{Z},
$$

and this allows to compute the spectrum of $\mathscr{A}_{\omega}$ as the union of spectra of the onedimensional spectral problems

$$
\sigma\left(\mathscr{A}_{\omega}\right)=\bigcup_{q \in \mathbb{Z}} \sigma\left(\mathscr{A}_{\omega, q}\right)
$$

where the operators $\mathscr{A}_{\omega, q}$ do not contain the angular variable.

\subsection{Three-dimensional Soler model}

In three spatial dimensions, it is convenient to consider equation (3) in spherical coordinates. We consider the 4-spinor solitary waves in the form of the Wakano Ansatz [155]:

$$
\psi(t, \mathbf{r})=\phi_{\omega}(\mathbf{r}) e^{-i \omega t}, \quad \phi_{\omega}(\mathbf{r})=\left[\begin{array}{c}
v(r, \omega)\left(\begin{array}{c}
1 \\
0
\end{array}\right) \\
i u(r, \omega)\left(\begin{array}{c}
\cos \theta \\
e^{i \varphi} \sin \theta
\end{array}\right)
\end{array}\right],
$$

with real-valued $v(r, \omega), u(r, \omega)$ satisfying

$$
\begin{aligned}
& \omega v=\left(\partial_{r}+\frac{2}{r}\right) u+\left[m-f\left(v^{2}-u^{2}\right)^{k}\right] v, \\
& \omega u=-\partial_{r} v-\left[m-f\left(v^{2}-u^{2}\right)^{k}\right] u .
\end{aligned}
$$

To study the linearization operator in the invariant space which has the same angular structure as the solitary waves, we consider the perturbed solutions in the form

$$
\psi(t, \mathbf{r})=\left[\begin{array}{c}
\left(v(r, \omega)+\xi_{1}(t, r)+i \eta_{1}(t, r)\right)\left(\begin{array}{c}
1 \\
0
\end{array}\right) \\
i\left(u(r, \omega)+\xi_{2}(t, r)+i \eta_{2}(t, r)\right)\left(\begin{array}{c}
\cos \theta \\
e^{i \varphi} \sin \theta
\end{array}\right)
\end{array}\right] e^{-i \omega t},
$$

with real-valued $\xi=\left[\xi_{1}, \xi_{2}\right]^{T} \in \mathbb{R}^{2}, \eta=\left[\eta_{1}, \eta_{2}\right]^{T} \in \mathbb{R}^{2}$ (note that the considered perturbation only depends on $r$ but not on the angular variables). The linearized equation on $R(t, r)=\left(\xi_{1}, \xi_{2}, \eta_{1}, \eta_{2}\right)^{T}$ is similar to equations (15), (16):

$$
\partial_{t} R=\mathscr{A}_{\omega} R \quad \text { with } \quad \mathscr{A}_{\omega}=\left[\begin{array}{cc}
0 & L_{-}(\omega) \\
-L_{+}(\omega) & 0
\end{array}\right],
$$


and

$$
\begin{aligned}
& L_{-}(\omega)=\left(\begin{array}{cc}
m-f(\tau)-\omega & \partial_{r}+\frac{2}{r} \\
-\partial_{r} & -m+f(\tau)-\omega
\end{array}\right), \\
& L_{+}(\omega)=L_{-}(\omega)-2 f^{\prime}(\tau)\left(\begin{array}{cc}
v^{2} & -v u \\
-v u & u^{2}
\end{array}\right),
\end{aligned}
$$

with $f(\tau)$ and $f^{\prime}(\tau)$ evaluated at $\tau \equiv v^{2}-u^{2}$.

\subsection{One-dimensional $\mathscr{P} \mathscr{T}$-symmetric Soler model}

To finish this section, we present a connection between the budding area of research of open $\mathscr{P} \mathscr{T}$-symmetric systems and the nonlinear Dirac equation. Open systems that feature a combination of gain and loss have become a focal point of numerous recent studies $[10,12,71,105]$. A principal reason for this was the introduction of the notion of $\mathscr{P} \mathscr{T}$-symmetry and its proposition by Bender (and collaborators) as an alternative to the postulate of Hermiticity in quantum mechanics. Its principal realization, though, came at the level of optical systems [95, 104, 133], where they were experimentally implemented in the context of waveguide arrays [77, 130,134]. Since this first set of efforts, numerous additional experiments emerged in the context of electronic circuits [136,137], in whispering-gallery microcavities [120], as well as in mechanical settings [11].

In Ref. [46], we introduced a generalized $\mathscr{P} \mathscr{T}$-symmetric 1D Soler model, which in covariant form reads as follows:

$$
\left(i \gamma^{\mu} \partial_{\mu}-m+g(\bar{\psi} \psi)^{k}+\gamma \gamma^{5}\right) \psi=0 .
$$

Alternatively, written in the standard form as a function of the bispinor components $\psi=\left[\psi_{1}(x), \psi_{2}(x)\right]^{T}$, the equations assume the following form:

$$
\begin{aligned}
& i \partial_{t} \psi_{1}=\partial_{x} \psi_{2}-g\left(\left|\psi_{1}\right|^{2}-\left|\psi_{2}\right|^{2}\right)^{k} \psi_{1}+m \psi_{1}+i \gamma \psi_{2}, \\
& i \partial_{t} \psi_{2}=-\partial_{x} \psi_{1}+g\left(\left|\psi_{1}\right|^{2}-\left|\psi_{2}\right|^{2}\right)^{k} \psi_{2}-m \psi_{2}+i \gamma \psi_{1} .
\end{aligned}
$$

Equations (20) are $\mathscr{P} \mathscr{T}$-symmetric due to their invariance under the transformation

$$
\mathscr{P}: x \rightarrow-x, \quad \psi_{1} \rightarrow \psi_{1}, \quad \psi_{2} \rightarrow-\psi_{2}
$$

and

$$
\mathscr{T}: t \rightarrow-t, \quad i \rightarrow-i, \quad \psi_{1} \rightarrow \psi_{1}, \quad \psi_{2} \rightarrow \psi_{2} .
$$

Here, we have assumed that $\psi_{1}(t, x)$ is even and $\psi_{2}(t, x)$ is odd. Comparing the present model to the standard Soler setting, we note the inclusion of the gain-loss 
term proportional to $\gamma$ through the Dirac matrix $\gamma^{5}$ (cf. [10]) multiplying the spinor $\psi$ in (19). For our two-component spinors, $\gamma^{5}$ is represented by the Pauli matrix $\sigma_{1}$ (1).

It is straightforward to see that in the linear case (of $g=0$ ), plane waves $\psi_{1}(t, x)=A e^{i(\kappa x-\omega t)}$ and $\psi_{2}(t, x)=i B e^{i(\kappa x-\omega t)}$ are solutions provided the dispersion relation $\omega= \pm \sqrt{m^{2}+\kappa^{2}-\gamma^{2}}$ is satisfied. Not only does the above formula have the characteristic Dirac form, but it also is consistent with the equivalence of the linear $\mathscr{P} \mathscr{T}$-Dirac equation with effective mass $\tilde{m}=\sqrt{m^{2}-\gamma^{2}}$, as per the above discussion.

In the present $\mathscr{P} \mathscr{T}$-NLD we have been unable to identify explicit stationary solitary waves in the massive $m \neq 0$ case, so we must rely on numerical analysis. In the same vein, it does not appear to be straightforward to generalize the transformation of $[10,13]$ to the present nonlinear setting.

To determine the stability of stationary solitary wave solutions, we consider infinitesimal perturbations of the form:

$$
\begin{aligned}
& \psi_{1}(t, x)=e^{-i \omega t}\left[v(x)+\delta\left(a_{1}(x) e^{\lambda t}+b_{1}^{*}(x) e^{\lambda^{*} t}\right)\right] \\
& \psi_{2}(t, x)=e^{-i \omega t}\left[u(x)+\delta\left(a_{2}(x) e^{\lambda t}+b_{2}^{*}(x) e^{\lambda^{*} t}\right)\right]
\end{aligned}
$$

where $\delta$ denotes a formal small parameter. The relevant linearization equations are derived to order $\mathrm{O}(\boldsymbol{\delta})$ [by substitution of the above Ansatz into Eqs. (20)] and are subsequently solved as a matrix eigenvalue problem

$$
\lambda\left[a_{1}(x), a_{2}(x), b_{1}(x), b_{2}(x)\right]^{T}=\mathscr{M}\left[a_{1}(x), a_{2}(x), b_{1}(x), b_{2}(x)\right]^{T},
$$

with $\mathscr{M}$ being

$$
\mathscr{M}=\left(\begin{array}{cc}
L_{1} & L_{2} \\
-L_{2}^{*} & -L_{1}^{*}
\end{array}\right)-i \gamma\left(\begin{array}{cc}
\gamma^{5} & 0 \\
0 & \gamma^{5}
\end{array}\right)
$$

and

$$
\begin{aligned}
L_{1} & =\left(\begin{array}{cc}
f\left(|v|^{2}-|u|^{2}\right)-m+\Lambda & -\partial_{x} \\
\partial_{x} & m-f\left(|v|^{2}-|u|^{2}\right)+\Lambda
\end{array}\right) \\
& +f^{\prime}\left(|v|^{2}-|u|^{2}\right)\left(\begin{array}{cc}
|v|^{2} & -v^{*} u \\
-v^{*} u & |u|^{2}
\end{array}\right) \\
L_{2} & =f^{\prime}\left(|v|^{2}-|u|^{2}\right)\left(\begin{array}{cc}
v^{2} & -v u \\
-v u & u^{2}
\end{array}\right) .
\end{aligned}
$$

From the dynamical equations (20) it is straightforward to show that the charge is not preserved. Instead, the following "moment equation" is satisfied: 


$$
\frac{d Q}{d t}=4 \gamma \int \operatorname{Re}\left(V^{*} U\right) d x
$$

Note that in the case of a standing wave state, $d Q / d t=0$ and charge is conserved.

Although the charge is not generally conserved, remarkably there is a conserved quantity in the form of the energy:

$$
E=\frac{1}{2} \int\left[\psi_{1}^{*} \partial_{x} \psi_{2}-\psi_{2}^{*} \partial_{x} \psi_{1}+m\left(\left|\psi_{1}\right|^{2}-\left|\psi_{2}\right|^{2}\right)-\frac{g}{k+1}\left[\left|\psi_{1}\right|^{2}-\left|\psi_{2}\right|^{2}\right]^{k+1}\right] d x .
$$

Notice the absence of a term proportional to $\gamma$ in this expression. Nevertheless, this energy is conserved not only for $\gamma=0$, but also for $\gamma \neq 0$, a feature that appears to be due to the use of the matrix $\gamma^{5}$ to introduce the $\mathscr{P} \mathscr{T}$-symmetry. As an additional observation associated with the unconventional nature of this system, we note that considering only the time-dependence and the terms proportional to $\gamma$, the resulting form reads: $i \partial_{t} \psi_{1}=i \gamma \psi_{2}$ and $i \partial_{t} \psi_{2}=i \gamma \psi_{1}$. This makes the two components appear as if they have both gain and loss.

Numerical results for this model can be found in [46].

\section{Solitary waves: exact solutions and numerical methods}

Solitary wave solutions of the form $\phi_{\omega}(x) e^{-i \omega t}, \omega \in(0, m)$, are known to exist in (3) and in other important systems based on the Dirac equation (see e.g. the review [58]). In the one-dimensional case, for pure power nonlinearity, the solutions are available in a closed form; see Section 3.1. However, for higher-dimensional cases, solitary wave and vortex solutions must be obtained by means of numerical methods. These methods can also be applied to 1D models with a general nonlinearity $f$ in (7) when the solutions are not available in a closed form.

\subsection{One-dimensional Soler model: exact solutions}

In [97] it was shown for the cubic nonlinearity, i.e. $k=1$ in (7), and later in [34, 41,111 ] for generic value $k>0$, that the solitary wave solutions can be found in a closed form for any $\omega \in(0, m)$ :

$$
\begin{aligned}
& v(x)=\cosh (k \beta x) \sqrt{\frac{(m+\omega)}{m+\omega \cosh (2 k \beta x)}}\left[\frac{(k+1) \beta^{2}}{m+\omega \cosh (2 k \beta x)}\right]^{1 / 2 k}, \\
& u(x)=\sinh (k \beta x) \sqrt{\frac{(m-\omega)}{m+\omega \cosh (2 k \beta x)}}\left[\frac{(k+1) \beta^{2}}{m+\omega \cosh (2 k \beta x)}\right]^{1 / 2 k},
\end{aligned}
$$

where $\beta=\sqrt{m^{2}-\omega^{2}}$. In the special case of $k=1$, waveforms in Eq. (22) reduce to 


$$
v(x)=\frac{\sqrt{2(m-\omega)}}{\left[1-\mu \tanh ^{2}(\beta x)\right] \cosh (\beta x)}, \quad u(x)=\frac{\sqrt{2 \mu(m-\omega)} \tanh (\beta x)}{\left[1-\mu \tanh ^{2}(\beta x)\right] \cosh (\beta x)},
$$

with $\mu=(m-\omega) /(m+\omega)$. Fig. 1 shows the profiles of solitary waves given by the expression (22) for $k=1$ and $k=3$. Notice that the first component of the spinor, $v(x)$, is spatially even, whereas the second component $u(x)$ is spatially odd. Moreover, $v^{2}(x)-u^{2}(x)>0$ for all $x \in \mathbb{R}$, so that the solitary waves satisfy the nonlinear Dirac equation (3) (with $n=1$ ) with both $f(s)=s$ and $f(s)=|s|$. Evaluating $\rho^{\prime \prime}$ at $x=0$, one can check that the charge density profiles $\rho(x)=\phi_{\omega}(x)^{*} \phi_{\omega}(x)$ (cf. (2.2)) become double-humped for $\omega \leq \omega_{h}(k)$, with $\omega_{h}(k)=m k /(k+1)$. The dependence of the charge and energy with respect to $\omega$ for different values of $k \in \mathbb{N}$ are shown in Fig. 2.

\subsection{Two-dimensional Soler model: numerical solutions}

No explicit solitary wave solutions are known for the Soler model in 2D (12). For this reason, one must rely on numerical results. We show in Section 3.2.1 the numerical methods used for the numerical determination of stationary solutions in (14). These methods can easily be adapted for numerically solving the Soler 3D model (18) (in the particular case of zero vorticity) and for finding solitary wave solutions in 1D models where additional terms to the equation (8) have been added, such as external fields [111] or terms of preserving $\mathscr{P} \mathscr{T}$ symmetry [45].

\subsubsection{Brief summary of spectral methods}

Prior to explaining the numerical methods used for calculating stationary solutions, we will proceed to present a summary of spectral methods needed for dealing with derivatives in continuum settings. For a detailed discussion on these methods, the reader is directed to [28] and references therein.

Spectral methods arise due to the necessity of calculating spatial derivatives with higher accuracy than that given by finite difference methods. As shown in [43], finite difference methods cannot be used for the stability and dynamics analysis of solitary waves in the Dirac equation.

In order to implement spectral derivatives, a differentiation matrix $\mathbf{D} \equiv\left\{D_{n, m}\right\}$ must be given together with $N$ collocation $^{2}$ (i.e. grid) points $\mathbf{x} \equiv\left\{x_{n}\right\}, n=1,2, \ldots N$, which are not necessarily equi-spaced. Thus, if the spectral derivative of a function $\mathbf{f}(\mathbf{x}) \equiv\left\{f_{n}\left(x_{n}\right)\right\}$ needs to be calculated, it can be cast as:

$$
f^{\prime}(x)=\partial_{x} f(x) \leftrightarrow f_{n}^{\prime}=\sum_{m=1}^{N} D_{n, m} f_{m},
$$

\footnotetext{
${ }^{2}$ Notice that this value of $N$ is not related to the dimension of the NLD, although the same symbol is used in both cases
} 

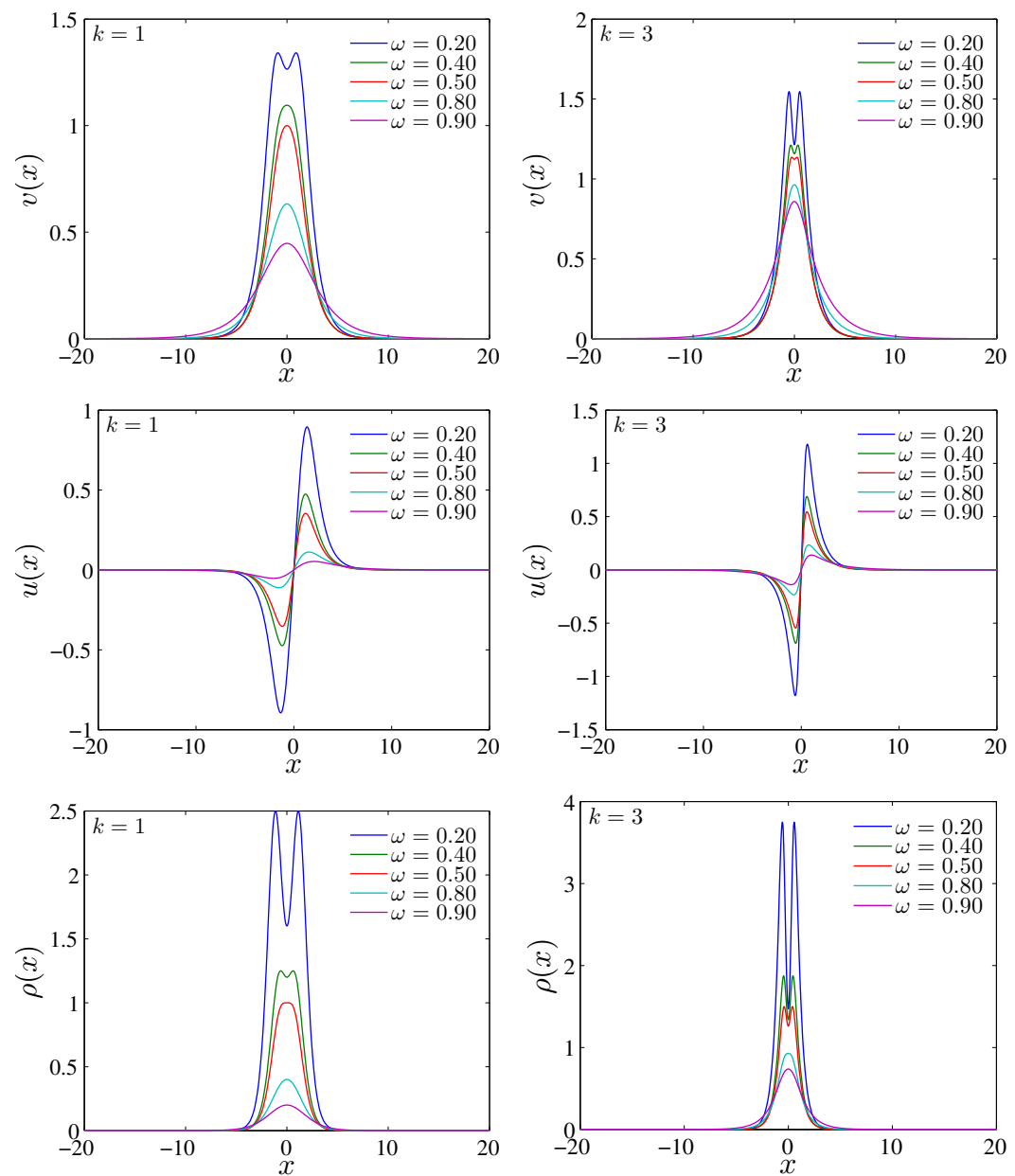

Fig. 1 Profile of solitary waves in the 1D Soler model. Figures depict the first and second spinor components together with the solitary wave density. Left (right) panels correspond to $k=1(k=3)$

where $f_{m} \equiv f\left(x_{m}\right)$ and $f_{n}^{\prime} \equiv f^{\prime}\left(x_{n}\right)$. If $x \in[-L, L]$ and the boundary conditions are periodic, the Fourier collocation can be used. In this case,

$$
x_{n}=\frac{2 L}{N}\left(n-\frac{N}{2}\right), \quad n=1,2, \ldots N
$$

with $N$ even. The differentiation matrix is 

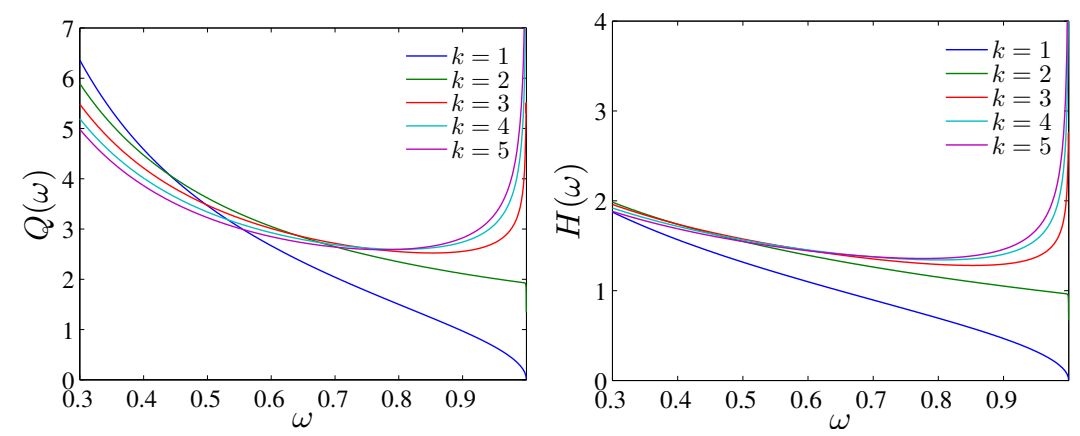

Fig. 2 Charge and energy of solitary waves (left and right panel, respectively) as functions of the frequency $\omega$ for $1 \leq k \leq 5$. Notice the existence of a minimum in the curve $Q(\omega)$ for $k>2$, which is related to the change in stability properties (see Section 5).

$$
D_{n, m}= \begin{cases}0 & \text { if } n=m, \\ \frac{\pi}{2 L} \frac{(-1)^{n+m}}{\tan \left[\left(x_{n}-x_{m}\right) / 2\right]} & \text { if } n \neq m .\end{cases}
$$

Notice that doing the multiplication Df is equivalent to performing the following pair of Discrete Fourier Transform applications:

$$
\mathbf{D f}=\mathscr{F}^{-1}(i \mathbf{k} \mathscr{F}(\mathbf{f})),
$$

with $\mathscr{F}$ and $\mathscr{F}^{-1}$ denoting, respectively, the direct and inverse discrete Fourier transform [150]. The vector wavenumber $\mathbf{k}=\left\{k_{n}\right\}$ is defined as:

$$
k_{n}= \begin{cases}\frac{n \pi}{L} & \text { if } n<N / 2, \\ 0 & \text { if } n=N / 2 .\end{cases}
$$

The computation of the direct and inverse discrete Fourier transforms, which is useful in simulations, can be accomplished by the Fast Fourier Transform. In what follows, however, the differentiation matrix is used for finding the Jacobian and stability matrices. Notice that the grid for a finite difference discretization is the same as in the Fourier collocation; and, in addition, there is a differentiation matrix for the finite difference method, i.e.

$$
D_{n, m}=\frac{1}{2 h}\left(\delta_{m, n+1}-\delta_{m, n-1}+\delta_{n, 1} \delta_{m, N}-\delta_{n, N} \delta_{m, 1}\right), \quad h=\frac{2 L}{N},
$$

with $\delta$ being Kronecker's delta. It can be observed from the above discussion that in the Fourier spectral method, the banded differentiation matrix of the finite difference method is substituted by a dense matrix, or, in other words, a nearest-neighbor interaction is exchanged with a long-range one. The lack of sparsity of differentia- 
tion matrices is one of the drawbacks of spectral methods, especially when having to diagonalize large systems. However, they have the advantage of needing (a considerably) smaller number of grid points $N$ for getting the same accuracy as with finite difference methods.

For fixed (Dirichlet) boundary conditions, the Chebyshev spectral methods are the most suitable ones. There are several collocation schemes, the Gauss-Lobatto being the most extensively used:

$$
x_{n}=L \cos \left(\frac{n \pi}{N+1}\right), \quad n=1,2, \ldots N,
$$

with $N$ being even or odd. The differentiation matrix is

$$
D_{n, m}= \begin{cases}\frac{x_{n}}{2 L\left(1-x_{n}^{2}\right)} & \text { if } n=m, \\ \frac{(-1)^{n+m}}{L \cos \left(x_{n}-x_{m}\right)} & \text { if } n \neq m .\end{cases}
$$

The significant drawback of Chebyshev collocation is that the discretization matrix possesses a great number of spurious eigenvalues [28]. They are approximately equal to $N / 2$. These spurious eigenvalues also have a significant non-zero real part, which increases when $N$ grows. This fact naturally reduces the efficiency of the method when performing numerical time-integration. However, it gives a higher accuracy than the Fourier collocation method when determining the spectrum of the stability matrix (see e.g. [43]).

Several modifications must be introduced when applying spectral methods to polar coordinates. They basically rely on overcoming the difficulty of not having Dirichlet boundary conditions at $r=0$ and the singularity of the equations at that point. In addition, in the case of the Dirac equation, the spinor components can be either symmetric or anti-symmetric in their radial dependence, so the method described in $[82,150]$ must be modified accordingly. As shown in the previously mentioned references, the radial derivative of a general function $f(r, \theta)$ can be expressed as:

$$
\partial_{r} f\left(r_{n}, \theta\right)=\sum_{m=1}^{N} D_{n, m} f\left(r_{m}, \theta\right)+D_{n, 2 N-m} f\left(r_{m}, \theta+\pi\right) .
$$

Notice that in this case, the collocation points must be taken as

$$
r_{n}=L \cos \left(\frac{n \pi}{2 N+1}\right), \quad n=1,2, \ldots 2 N,
$$

but only the first $N$ points are taken so that the domain of the radial coordinate does not include $r=0$. Analogously the differentiation matrix would possess now $2 N \times 2 N$ components, but only the upper half of the matrix, of size $N \times 2 N$ is used.

If the function that must be derived is symmetric or anti-symmetric, i.e. $f(r, \theta+$ $\pi)= \pm f(r, \theta)$, with the upper (lower) sign corresponding to the (anti-)symmetric function, equation (26) can be written as follows: 


$$
\partial_{r} f\left(r_{n}, \theta\right)=\sum_{m=1}^{N}\left[\left(D_{n, m} \pm D_{n, 2 N-m}\right) f\left(r_{m}, \theta\right)\right] .
$$

Thus, the differentiation matrix has a different form depending on whether $f(r, \theta)$ is symmetric or anti-symmetric:

$$
\partial_{r} \mathbf{f}(\mathbf{r}, \theta)=\mathbf{D}^{( \pm)} \mathbf{f} \quad \text { if } f(r, \theta)= \pm f(r, \theta+\pi)
$$

with $\mathbf{r} \equiv\left\{r_{n}\right\}, \mathbf{f}(\mathbf{r}) \equiv\left\{f\left(r_{n}\right)\right\}$ and $\mathbf{D}^{( \pm)} \mathbf{f}$ defined as in (27).

\subsubsection{Fixed point methods}

Among the numerical methods available for solving nonlinear systems of equations we have chosen to use fixed point methods, such as the Newton-Raphson one [123], which requires the transformation of the set of two coupled ordinary differential equations (14) into a set of $2 N$ algebraic equations; this is performed by defining the set of collocation points $\mathbf{r} \equiv\left\{r_{n}\right\}$, and transforming the derivatives into multiplication of the differentiation matrices $\mathbf{D}^{(1)}$ and $\mathbf{D}^{(2)}$ (to be defined below) times the vectors $\mathbf{u} \equiv\left\{u_{n}\right\}$ and $\mathbf{v} \equiv\left\{v_{n}\right\}$, respectively, being $u_{n} \equiv u\left(r_{n}\right)$ and $v_{n} \equiv v\left(r_{n}\right)$ as explained in the previous section. Thus, the discrete version of (14) reads:

$$
\begin{aligned}
& F_{n}^{(1)} \equiv(m-\omega) v_{n}-g \tau_{n}^{k} v_{n}+\sum_{m} D_{n m}^{(2)} u_{m}+\frac{S+1}{r_{n}} u_{n}=0, \\
& F_{n}^{(2)} \equiv(m+\omega) u_{n}-g \tau_{n}^{k} u_{n}+\sum_{m} D_{n m}^{(1)} v_{m}+\frac{S}{r_{n}} v_{n}=0,
\end{aligned}
$$

with $\tau_{n} \equiv v_{n}^{2}-u_{n}^{2}$. It is important to notice that matrices $\mathbf{D}^{(1)}$ and $\mathbf{D}^{(2)}$ correspond to either $\mathbf{D}^{(+)}$or $\mathbf{D}^{(-)}$, depending on the symmetry of $\mathbf{v}$ and $\mathbf{u}$, which, at the same time, depend on the value of the vorticity $S$. If $S$ is even, then $\mathbf{v}$ and $\mathbf{u}$ are symmetric and antisymmetric, respectively, being $\mathbf{D}^{(1)}=\mathbf{D}^{(+)}$and $\mathbf{D}^{(2)}=\mathbf{D}^{(-)}$. On the contrary, if $S$ is odd, then $\mathbf{u}$ is symmetric and $\mathbf{v}$ is antisymmetric, being $\mathbf{D}^{(1)}=\mathbf{D}^{(-)}$and $\mathbf{D}^{(2)}=\mathbf{D}^{(+)}$.

In order to find the roots of the vector function $\mathbf{F}=\left(\left\{F_{n}^{(1)}\right\},\left\{F_{n}^{(2)}\right\}\right)^{T}$, an analytical expression of the Jacobian matrix

$$
\mathbf{J}=\left(\begin{array}{cc}
\frac{\partial \mathbf{F}^{(\mathbf{1})}}{\partial \mathbf{u}} & \frac{\partial \mathbf{F}^{(\mathbf{1})}}{\partial \mathbf{v}} \\
\frac{\partial \mathbf{F}^{(2)}}{\partial \mathbf{u}} & \frac{\partial \mathbf{F}^{(2)}}{\partial \mathbf{v}}
\end{array}\right)=\left(\begin{array}{cc}
(m-\omega)-g \tau^{k-1}\left[2 k v^{2}+\tau\right] & 2 k g \mathbf{v u} \tau^{k-1}+D^{(2)}+\frac{S+1}{r} \\
-2 k g \mathbf{v u} \tau^{k-1}+D^{(1)}-\frac{S}{r} & (m+\omega)-g \tau^{k-1}\left[\tau-2 k v^{2}\right]
\end{array}\right)
$$

must be introduced, with the derivatives expressed by means of spectral methods and the matrix is evaluated at the corresponding grid points. The roots of $\mathbf{F}, \Phi=$ $(\mathbf{v}, \mathbf{u})^{T}$, are found by successive application of the iteration $\Phi \rightarrow \Phi-\mathbf{J}^{-1} \mathbf{F}$ until 
convergence is attained. In our case, we have chosen as convergence condition that $\|\mathbf{F}\|_{\infty}<10^{-10}$.

Spectral stability is analyzed by evaluating the functions appearing in matrix $\mathscr{A}_{\omega}$ of equation (16) at the collocation points and substituting the partial derivatives by the corresponding differentiation matrices. At this point, one must be very cautious because, as also occurred with the Jacobian, there will be two different differentiation matrices in our problem. Now $L_{-}(\omega)$ will be represented by the following matrix:

$$
L_{-}(\omega)=\left(\begin{array}{cc}
f(\tau)-\omega & D^{(2)}+\frac{S+1}{r} \\
-\left(D^{(1)}-\frac{S}{r}\right)-f(\tau)-\omega
\end{array}\right) .
$$

\subsubsection{Solitary waves and vortices}

This section deals with the numerically found profiles for solitary waves $(S=0)$ and vortices $(S=1)$ in the two-dimensional Soler model. Fig. 3 shows, in radial coordinates, the profiles of each component of $S=0$ solitary waves with $k=1$ and $k=2$; the left panels of Fig. 4 depict those components for $S=1$ vortices. As explained in Section 3.2.1, the first spinor component is spatially symmetric whereas the second component is anti-symmetric as long as the vorticity of the first component, $S \in \mathbb{Z}$, is even. The spatial symmetry is inverted if $S$ is odd. Notice also that in the $S=0$ case, the solution profile has a hump for $r>0$ whenever $\omega$ is below a critical value. It manifests as the transformation of the solitary wave density from a circle to a ring. The ring radius increases when $\omega$ decreases, becoming infinite when $\omega \rightarrow 0$. For this reason, computations are progressively more demanding for smaller values of $\omega$.

The right panels of Fig. 4 show the radial profile of $S=0$ solitary waves in 3D. We have not included solitary waves with higher vorticity because, as explained in Subsection 2.5, the Soler equation in radial coordinates can only be expressed in the $S=0$ case. Fig. 5 shows the charge for the $S=0$ solitary waves in the 2D and 3D Soler models for different values of $k$.

It is worth mentioning that, despite the absence of an explicit analytical form of $3 \mathrm{D}$ solitary waves, their existence has been rigorously proven in [33, 59, 109].

\section{Stability of solitary waves: theoretical results}

In Section 2, we presented the equation governing the linear stability analysis of stationary solutions. In the present section, we will show the theoretical background related to spectral and orbital stability. Many of the results proposed herein will be numerically checked in Section 5 . 

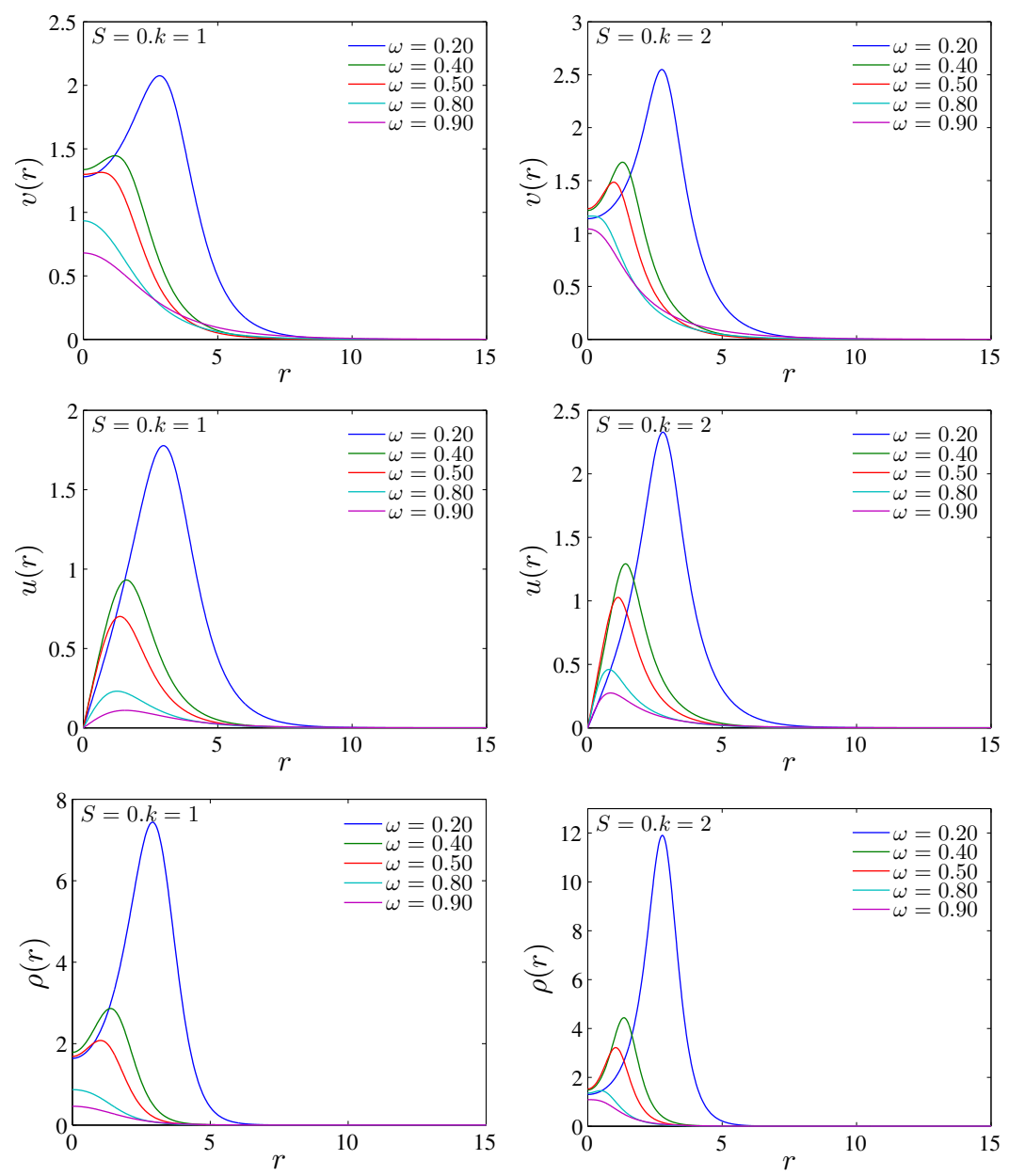

Fig. 3 Radial profile of $S=0$ solitary waves in the 2D Soler model. Figures depict the first and second spinor components together with the solution density. Left (right) panels correspond to $k=1(k=2)$.

\subsection{Spectral stability of solitary waves}

Prior to proceeding to the spectral stability analysis, we introduce some definitions.

The linearization of (3) at a solitary wave solution $\psi(t, x)=\phi_{\omega}(x) e^{-i \omega t}$ is represented by non-self-adjoint operators of the form

$$
J\left(D_{m}-\omega+V(x, \omega)\right), \quad \text { with } J \text { skew-adjoint, } J^{2}=-1,
$$



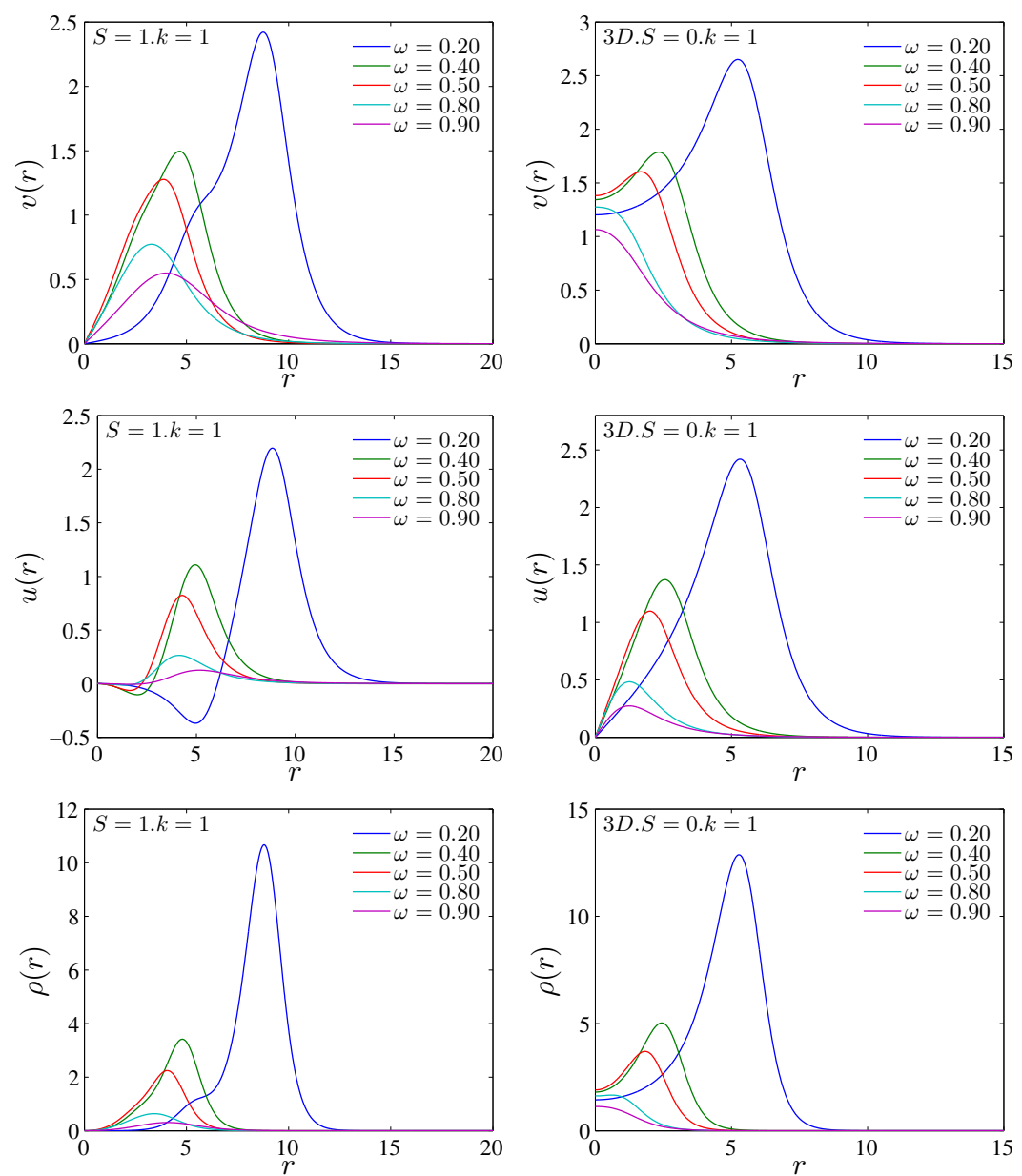

Fig. 4 (Left panels) Radial profile of $S=1$ vortices in the cubic 2D Soler model. (Right panels) Radial profile of $S=0$ solitary waves in the cubic 3D Soler model. Figures depict the first and second spinor components together with the solution density.

where the matrix $J$ commutes with $D_{m}$ but not necessarily with the potential $V(x, \omega)$.

We say that the solitary wave is spectrally stable if the spectrum of its linearization operator has no points with positive real part. The spectral stability is the weakest type of stability; it does not necessarily lead to actual, dynamical one. The essential spectrum is easy to analyze: the application of Weyl's theorem (see e.g. [129, Theorem XIII.14, Corollary 2] ) shows that the essential spectrum of the operator corresponding to the linearization at a solitary wave starts at $\pm(m-|\omega|) i$ and extends to $\pm \infty i$. Thus, the spectral stability of the corresponding solitary wave would 

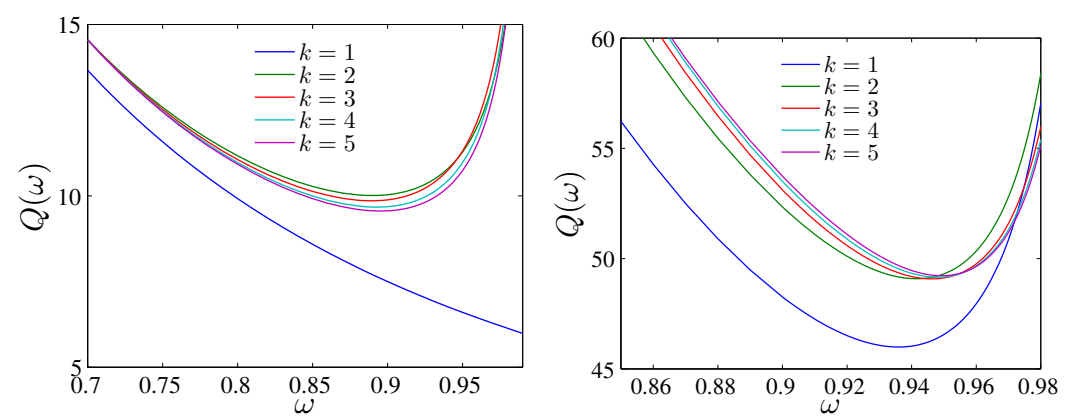

Fig. 5 Dependence of the charge of $S=0$ solitary waves in the 2D and 3D Soler models (left and right panels, respectively) with respect to the frequency for $1 \leq k \leq 5$. Notice the existence of a minimum in the 2D (3D) curve for $k>1(k \geq 1)$, which will be related to stability changes (see Section 5).

be a corollary of the absence of eigenvalues with positive real part in the spectrum of $J\left(D_{m}-\omega+V(\omega)\right)$ in (28). The major difficulties in identifying the point spectrum $\sigma_{p}\left(J\left(D_{m}-\omega+V(\omega)\right)\right)$ are due to the spectrum of $D_{m}$ extending to both $\pm \infty$; this prevents us from using standard tools developed in the NLS context.

In the absence of linear stability (that is when the linearized system is not dynamically stable), one expects to be able to prove orbital instability, in the sense of [74]; in [73], such instability is proved in the context of the nonlinear Schrödinger equation; such results are still absent for the nonlinear Dirac equation.

Since the isolated eigenvalues depend continuously on the perturbation, it is convenient to trace the location of "unstable" eigenvalues (eigenvalues with positive real part) considering $\omega$ as a parameter. One wants to know how and when the "unstable" eigenvalues may emerge from the imaginary axis, particularly from the essential spectrum; that is, at which critical values of $\omega$ the solitary waves start developing an instability. Below, we describe the possible scenarios.

\section{Instability scenario 1: collision of eigenvalues}

The well-known Vakhitov-Kolokolov stability criterion [151] keeps track of the collision of purely imaginary eigenvalues at the origin and a subsequent birth of a positive and a negative eigenvalue. This criterion was discovered in the context of nonlinear Schrödinger equations, in relation to ground state solitary waves $\phi_{\omega}(x) e^{-i \omega t}$ ("ground state" in the sense that $\phi_{\omega}(x)$ is strictly positive; for more details, see [14]). When $\partial_{\omega} Q(\omega)<0$, with $Q(\omega)=\left\|\phi_{\omega}\right\|_{L^{2}}^{2}$ being the charge of the solitary wave (2.2), then the linearization at a solitary wave has purely imaginary spectrum; when $\partial_{\omega} Q(\omega)>0$, there are two real (one positive, one negative) eigenvalues of the linearization operator. The vanishing of the quantity $\partial_{\omega} Q(\omega)$ at some 
Fig. 6 Birth of "unstable" eigenvalues out of collisions of imaginary eigenvalues. When the frequency $\omega$ of the solitary wave $\phi_{\omega} e^{-i \omega t}$ changes, the "unstable", positive-real-part eigenvalues in the linearized equation could be born from the collisions of discrete imaginary eigenvalues

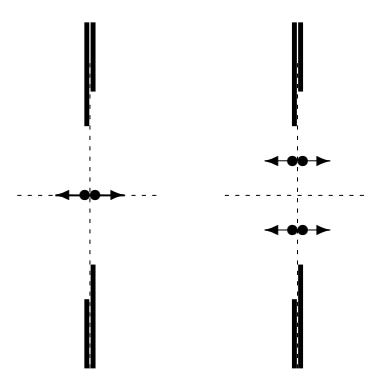

value of $\omega$ indicates the moment of the collision of eigenvalues, when the Jordan block corresponding to the zero eigenvalue has a jump of two in its size. A nice feature of the linearization at a ground state solitary wave in the nonlinear Schrödinger equation is that its spectrum belongs to the imaginary axis, with some eigenvalues possibly located on the real axis; thus, the collision of eigenvalues at $\lambda=0$ is the only way the spectral instability could develop. In the NLD context, such a collision does not necessarily occur at $\lambda=0$; both situations as in Fig. 6 are possible.

In [16], it was shown that in NLD (and similar fermionic systems) the collision of eigenvalues at the origin and a subsequent transition to instability is characterized not only by the Vakhitov-Kolokolov condition $d Q / d \omega=0$, but also by the condition $E(\omega)=0$, where $E$ is the value of the energy functional on the corresponding solitary wave.

Theorem 1. The algebraic multiplicity of the eigenvalue $\lambda=0$ of the linearization $\mathscr{A}_{\omega}$ at the solitary wave $\phi_{\omega}(x) e^{-i \omega t}$ has a jump of (at least) 2 when at a particular value of $\omega$ either $\partial_{\omega} Q\left(\phi_{\omega}\right)=0$ or $E\left(\phi_{\omega}\right)=0$, with $Q\left(\phi_{\omega}\right)$ and $E\left(\phi_{\omega}\right)$ being the charge and the energy of the solitary wave $\phi_{\omega}(x) e^{-i \omega t}$.

The eigenvalues with positive real part could also be born from the collision of purely imaginary eigenvalues at some point in the spectral gap but away from the origin; we have recently observed this scenario in the cubic Soler model in two spatial dimensions [46]. Presently we do not have a criterion for such a collision of eigenvalues.

\section{Instability scenario 2: bifurcations from the essential spectrum}

The most peculiar feature of the linearization at a solitary wave in the NLD context is the possibility of bifurcations of eigenvalues with nonzero real part off the imaginary axis, out of the bulk of the essential spectrum.

The article [25] gives a thorough analytical study of eigenvalues of the Dirac operators, focusing on whether and how such eigenvalues can bifurcate from the essential spectrum. Generalizing the Jensen-Kato approach [85] to the context of the Dirac operators, it was shown in [25, Theorem 2.15] that for $|\omega|<m$ the bifurcations from the essential spectrum are only possible from embedded eigenvalues 
Fig. 7 Possible bifurcations from the essential spectrum. Theoretically, when $|\omega|<m$, the nonzero-real-part eigenvalues could be born from the embedded thresholds at $\pm i(m+|\omega|)$, from the embedded eigenvalue in the bulk of the essential spectrum between the threshold and the embedded threshold, and from the collision of the thresholds at \pm im when $\omega=0$.

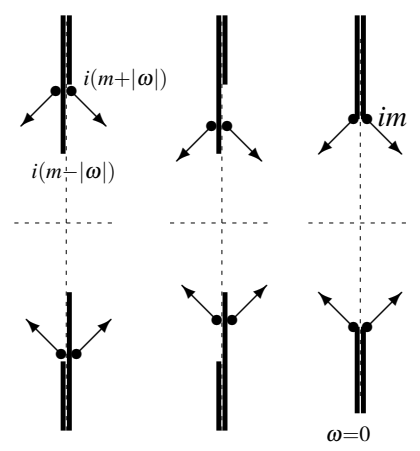

(Fig. 7, center), with the following exceptions: the bifurcation could start at the embedded thresholds located at $\pm i(m+|\omega|)$ (Fig. 7, left), or they could start at $\lambda= \pm i m$ when $\omega=0$ (Fig. 7, right; this situation correspond to the collision of thresholds). Indeed, bifurcations from the embedded thresholds have been observed in a onedimensional NLD-type model of coupled-mode equations [9,34]. The bifurcations from the collision of thresholds at $\pm i m$ (when $\omega=0$ ) were demonstrated in [88] in the context of the perturbed massive Thirring model.

One can use the Carleman-Berthier-Georgescu estimates [17] to prove that there are no embedded eigenvalues (hence no bifurcations) in the portion of the essential spectrum outside of the embedded thresholds [25].

As to the bifurcations from the embedded eigenvalues before the embedded thresholds, as in Fig. 7 (center), we do not have any such examples in the NLD context, although such examples could be produced for Dirac operators of the form (28) (with $V$ kept self-adjoint).

\section{Instability scenario 3: bifurcations from the nonrelativistic limit}

Fig. 8 Bifurcations from $\lambda=0$ and hypothetical bifurcations from $\lambda= \pm 2 m i$ in the nonrelativistic limit, $\omega \lesssim m$. The nonzero-real-part eigenvalues could be present in the spectrum of the linearization at a solitary wave $\phi_{\omega} e^{-i \omega t}$ for $\omega$ arbitrarily close to $m$; these eigenvalues would have to be located near $\lambda=0$ or near the embedded threshold at $\lambda= \pm 2 m i$.

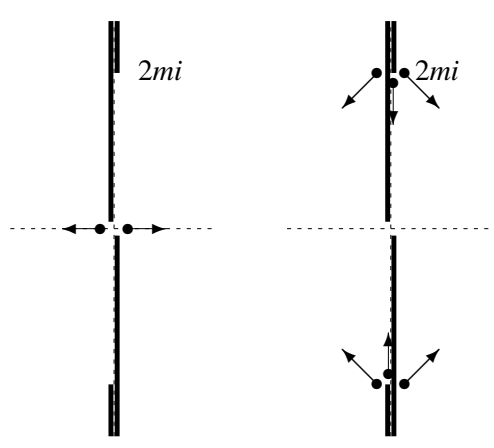


The nonzero-real-part eigenvalues could be present in the spectrum of the linearization operators at small amplitude solitary waves for all $\omega \lesssim m$, being born "from the nonrelativistic limit". It was proved in [25, Theorem 2.19], under very mild assumptions, that the bifurcations of eigenvalues for $\omega$ departing from $\pm m$ are only possible from the thresholds $\lambda=0$ and $\lambda= \pm 2 m i$; see Fig. 8 .

We now undertake a detailed study of these bifurcations; let us concentrate on the case $\lambda=0$. It is of no surprise that the behaviour of eigenvalues of the linearized operator near $\lambda=0$, in the nonrelativistic limit $\omega \lesssim m$, follows closely the pattern which one observes in the nonlinear Schrödinger equation with the same nonlinearity. In other words, if the linearizations of the nonlinear Dirac equation at solitary waves with $\omega \lesssim m$ admit a family of eigenvalues $\Lambda_{\omega}$ which continuously depends on $\omega$, such that $\Lambda_{\omega} \rightarrow 0$ as $\omega \rightarrow m$, then this family is merely a deformation of an eigenvalue family $\Lambda_{\omega}^{\mathrm{NLS}}$ of the linearization of the nonlinear Schrödinger equation with the same nonlinearity (linearized at corresponding solitary waves). To make this rigorous, one considers the spectral problem for the linearization at a solitary wave with $\omega \lesssim m$, applies the rescaling with respect to $m-\omega \ll 1$, and uses the reduction based on the Schur complement method, recovering in the nonrelativistic limit $\omega \rightarrow m$ the linearization of the nonlinear Schrödinger equation, and then applying the Rayleigh-Schrödinger perturbation theory; in [36], this approach was developed to prove the linear instability of small amplitude solitary waves $\phi_{\omega}(x) e^{-i \omega t}$ in the "charge-supercritical" NLD, in the nonrelativistic limit $\omega \lesssim m$.

Theorem 2. Assume that $f(s)=|s|^{k}$, where $k \in \mathbb{N}$ satisfies $k>2 / n$ (and $k<2$ for $n=3$ ). Then there is $\omega_{1}<m$ such that the solitary wave solutions $\phi_{\omega}(x) e^{-i \omega t}$ (in the form of the Wakano Ansatz (2.5)) to NLD are linearly unstable for $\omega \in\left(\omega_{1}, m\right)$. More precisely, let $\mathscr{A}_{\omega}$ be the linearization of the nonlinear Dirac equation at a solitary wave $\phi_{\omega}(x) e^{-i \omega t}$. Then for $\omega \in\left(\omega_{1}, m\right)$ there are eigenvalues

$$
\pm \lambda_{\omega} \in \sigma_{p}\left(\mathscr{A}_{\omega}\right), \quad \lambda_{\omega}>0, \quad \lambda_{\omega}=O(m-\omega)
$$

Let us remark here that the restriction in the above theorem that $k$ is a natural number which was needed to make sure that the solitary wave family of the form of the Wakano Ansatz indeed exists. Theorem 2 extends to $f(s)=a|s|^{k}+O\left(|s|^{K}\right)$, $a>0$, with $k \in(2 / n, 2 /(n-2))(k>2 / n$ when $n \leq 3)$ and $K>k$. The existence of the corresponding families of solitary waves was later proved in [24]. In that article, a general construction was given for small amplitude solitary waves in the nonlinear Dirac equation, deriving the asymptotics which we will need in the forthcoming stability analysis of such solitary waves. This is a general result proved for nonlinearities which are not necessarily smooth, thus applicable to e.g. critical and subcritical nonlinearities.

We point out that the instability stated in Theorem 2 is in a formal agreement with the Vakhitov-Kolokolov stability criterion [151]; one has $d Q(\omega) / d \omega>0$ for $\omega \lesssim m$. Conversely, we expect that the presence of eigenvalues with nonzero real part in the vicinity of $\lambda=0$ for $\omega \lesssim m$, is prohibited by the Vakhitov-Kolokolov stability criterion $\frac{d Q(\omega)}{d \omega}<0, \omega \lesssim m$. 
Similarly to how the NLS corresponds to the nonrelativistic limit of NLD, in the nonrelativistic limit of the Dirac-Maxwell system one arrives at the Choquard equation [99]; see [38] and the references therein. The Choquard equation is known to be spectrally (in fact, even orbitally) stable [32]; we expect that this implies absence of unstable eigenvalues bifurcating from the origin in the Dirac-Maxwell system.

As we pointed out above, in the nonrelativistic limit $\omega \lesssim m$, there could be eigenvalue families of the linearization of the nonlinear Dirac operator bifurcating not only from the origin, but also from the embedded threshold (that is, such that $\left.\lim _{\omega \rightarrow m} \Lambda_{i}(\omega)= \pm 2 m i\right)$. Rescaling and using the Schur complement approach shows that there could be at most $N / 2$ such families bifurcating from each of $\pm 2 m i$, with $N$ the number of components of a spinor field (in 3D Dirac, one takes $N=4$ ). Could these eigenvalues go off the imaginary axis into the complex plane? While for the nonlinear Dirac equations with a general nonlinearity the answer to this question is unknown, in the Soler model we can exclude this scenario. One can show that there are exact eigenvalues $\lambda_{ \pm}(\omega)= \pm 2 \omega i$, each being of multiplicity $N / 2$; thus, we know exactly what happens to the eigenvalues which bifurcate from $\pm 2 m i$, and expect no bifurcations of eigenvalues off the imaginary axis. The details are given in [26].

Let us finish with a very important result: the existence of eigenvalues $\pm 2 \omega i$ of the linearization at a solitary wave in the Soler model (3) is a consequence of having bi-frequency solitary wave solutions in the Soler model, in any dimension and for any nonlinearity. For more details, see [26].

\subsection{Orbital and asymptotic stability of solitary waves}

The spectral analysis is one aspect of global analysis of the dynamical stability. In principle any spectral instability around a stationary solution should lead to a dynamical instability, namely the stationary solution is orbitally unstable. The contrapuntal statement that a stable stationary state has a spectrally stable linearized operator needs to be analyzed carefully.

If the Dirac operator $D_{m}$ is perturbed by some zero-order external potential, the perturbation theory provides tools which allow one to analyze the linear stability of linearized operators of the form (28). Still some important restrictions on the potential appear (decay, regularity, and absence of resonances). Even if the perturbation analysis needs some work, it is much less involved compared to the complete spectral characterization of the linearized operator. This opens the gates to the analysis of the nonlinear stability.

Prior to a bibliographical review of the available works in this direction, we make a remark. While in many models the orbital stability is obtained by using the energy as some kind of a Lyapunov functional, this is no longer possible for models of Dirac type since the energy is sign-indefinite. Even if there are some conserved quantities which allow one to control certain negative directions of the Hessian of the energy, 
the latter are in infinite number ("infinite Morse index") and in most cases the conservation laws are not enough. The route "use linear stability to prove the asymptotic stability" seems to be the only one available for the sign-indefinite systems such as nonlinear Dirac, Dirac-Hartree-Fock, and others. As a result, due to the strong indefiniteness of the Dirac operator (the energy conservation does not lead to any bounds on the $H^{1 / 2}$-norm), we do not know how to prove the orbital stability [74] but via proving the asymptotic stability first. The only exceptional case in nonlinear Dirac-type systems seems to be the completely integrable massive Thirring model in one spatial dimension [149], where additional conserved quantities arising from the complete integrability allow one to prove orbital stability of solitary waves $[40,118]$. Note that these conserved quantities are used not to control the negative directions but rather to construct a new Lyapunov functional. More precisely, by [118], there is a functional $R$ defined on $H^{1}\left(\mathbb{R}, \mathbb{C}^{2}\right.$ ) (which contains terms dependent on powers of components of $\psi \in \mathbb{C}^{2}$ of order up to six) which is (formally) conserved for solutions to the massive Thirring model, and it is further shown that there is $\omega_{0} \in(0, m]$ such that for $\omega \in\left(-\omega_{0}, \omega_{0}\right)$ the solitary wave amplitude is a local minimizer of $R$ in $H^{1}$ under the charge and momentum conservation, and hence the corresponding solitary wave is orbitally stable in $H^{1}\left(\mathbb{R}, \mathbb{C}^{2}\right)$. Moreover, in [40], using the global existence of $L^{2}$-solutions for the (cubic) massive Thirring model [31], the orbital stability of solitary waves in $L^{2}(\mathbb{R})$ has been shown, with the proof based on the auto-Bäcklund transformation. Now we turn to the asymptotic stability. In [37], the asymptotic stability was proved for the small energy perturbations to solitary waves in the Gross-Neveu model. The model is taken with particular pure-power nonlinearities when all the assumptions on the spectral and linear stability of solitary waves have been verified directly. This is, referring to the previous discussion, also the "proof of concept": it is shown that there are translation-invariant systems based on the Dirac operator which are asymptotically stable; this is in spite of the energy functional being unbounded from below.

First results on asymptotic stability were obtained in [22,23] in the case $n=3$, in the external potential. There, the spectrum of the linear part of the equation $D_{m}+V$ is supposed to be, beside the essential spectrum $\mathbb{R} \backslash(-m, m)$, formed by two simple eigenvalues; let us denote them by $\lambda_{0}$ and $\lambda_{1}$, with $\lambda_{0}<\lambda_{1}$. From the associated eigenspaces, there is a bifurcation of small solitary waves for the nonlinear equation. The corresponding linearized operators are exponentially localized small perturbations of $D_{m}+V$, so that the perturbation theory allows a precise knowledge of the resulting spectral stability. Depending on the distance from $\lambda_{0}$ to $\lambda_{1}$ compared to the distance from $\lambda_{0}$ to the essential spectrum, the resulting point spectrum for the linearized operator may be discrete and purely imaginary and hence spectrally stable, or instead it may have nonzero-real-part eigenvalues if a "nonlinear Fermi Golden Rule" assumption is satisfied (similarly to the Schrödinger case, see $[30,141,142])$; in the latter case, linear and dynamical instabilities occur. In the former case, the linear stability follows from the spectral one via the perturbation theory. In any case, using the dispersive properties for perturbations of $D_{m}$, there is a stable manifold of real codimension 2. Due to the presence of nonzero discrete modes, even in the linearly stable case, the dynamical stability is not guaranteed. 
Before considering the results on the dynamics outside this manifold, for perturbations along the remaining two real directions, one could ask what might happen if $D_{m}+V$ has only one eigenvalue. The answer follows quite immediately with the ideas from $[22,23]$. In this case, there is only one family of solitary waves and it is asymptotically stable. Notice that the asymptotic profile is possibly another solitary wave but close to the perturbed one. In the one-dimensional case, this was studied properly in [118]. Note that the one-dimensional framework suffers from relatively weak dispersion which makes the analysis of the stabilization process more delicate. As for the dynamics outside the above-mentioned stable manifold, the techniques rely on the analysis of nonlinear resonances between discrete isolated modes and the essential spectrum where the dispersion takes place. This requires the normal form analysis in order to isolate the leading resonant interactions. The former is possible only if the "nonlinear Fermi Golden Rule" is imposed. Such an analysis was done in [15] but in a slightly different framework: instead of considering the perturbative case the authors chose the translation-invariant case, imposing a series of assumptions that lead to the spectral stability of solitary waves. These assumptions are verified in some perturbative context with $V \neq 0$. This case is analyzed in [42]. The asymptotic stability approach from $[15,37,118]$ is developed under important restrictions on the types of admissible perturbations. These restrictions are needed to avoid the translation invariance and, most importantly, to prohibit the perturbations in the direction of exceptional eigenvalues $\pm 2 \omega i$ of the linearization operator at a solitary wave $\phi_{\omega}(x) e^{-i \omega t}$. These eigenvalues are a feature of the Soler model (see $[49,72]$ ); they are present in the spectrum for any nonlinearity $f$ in the Soler model (3), see [27, 49,72]. These eigenvalues are embedded into the essential spectrum when $|\omega|>m / 3$ and violate the "nonlinear Fermi Golden Rule": they do not "interact" (that is, do not resonate) with the essential spectrum; the energy from the corresponding modes does not disperse to infinity. This does not allow the standard approach to proving the asymptotic stability.

\section{Stability of solitary waves: numerical results}

Once the theoretical background on linear stability has been presented, we review in this section some very recent numerical results on this topic. To this aim, we first include a brief introduction to the Evans function formalism [63], and then, detailed results based on numerical analysis of BdG-like spectral stability are shown for both 1D and 2D Soler models.

Let us recall some notation regarding the spectral stability, as we will make an extensive use of them in what follows. The essential spectrum corresponds to $\lambda \in$ $i(-\infty,|\omega|-m] \cup i[-|\omega|+m, \infty)$. Embedded eigenvalues can be in the region $\lambda \in$ $\pm i[-|\omega|+m,|\omega|+m]$ of the essential spectrum; for abbreviation, we denote this region as the embedded spectrum and the remaining part of the essential spectrum as non-embedded spectrum. 
In what follows, without lack of generality we will take $g=m=1$ unless stated otherwise.

\subsection{Evans function approach to the analysis of spectral stability}

The study of the spectral stability of the cubic 1D Soler model was performed in [27], with the aid of the Evans function technique. This was the first definitive linear stability result (as well as the first definite stability result) in the context of the nonlinear Dirac equation.

Let us give more details. In order to compute $\sigma\left(\mathscr{A}_{\omega}\right)$ we can employ the Evans function which provides an efficient tool to locate the point spectrum. The Evans function was first introduced by J.W. Evans [63-66] in his study of the stability of nerve impulses. In his work, Evans defined $D(\lambda)$ to represent the determinant of eigenvalue problems associated with traveling waves of a class of nerve impulse models. $D(\lambda)$ was constructed to detect the intersections of the subspace of solutions decaying exponentially to the right and the subspace of solutions decaying exponentially to the left. Jones [87] used Evans' idea to study the stability of a singularly perturbed FitzHugh-Nagumo system. Jones called it the Evans function, and the notation $E(\lambda)$ is now common. The first general definition of the Evans function was given by Alexander et al. [6] in their study of the stability for traveling waves of a semi-linear parabolic system. Pego and Weinstein [114] expanded on Jones' construction of Evans function to study the linear instability of solitary waves in the Korteweg-de Vries equation (KdV), the Benjamin-Bona-Mahoney equation $(\mathrm{BBM})$, and the Boussinesq equation. Generally, the Evans function for a differential operator $\mathscr{D}$ is an analytic function such that $E(\lambda)=0$ if and only if $\lambda$ is an eigenvalue of $\mathscr{D}$, and the order of zero is equal to the algebraic multiplicity of the eigenvalue.

Let us give a simple example which illustrates the nature of the Evans function. Consider the stationary Schrödinger equation

$$
-\lambda^{2} u(x)=H u(x)
$$

where $H=-\partial_{x}^{2}+V$ with $V \in C(\mathbb{R}), \operatorname{supp}(V) \subset(-1,1)$. For $\lambda \in \mathbb{C} \backslash\{0\}, \operatorname{Re}(\lambda)>0$, it has the solutions $J_{+}(\lambda, x)$ and $J_{-}(\lambda, x), x \in \mathbb{R}$, defined by their behaviour at $\pm \infty$ :

$$
J_{+}(\lambda, x)=e^{-\lambda x}, \quad x \geq 1 ; \quad J_{-}(\lambda, x)=e^{+\lambda x}, \quad x \leq-1 .
$$

We should note that $J_{+}$and $J_{-}$decay exponentially as $x \rightarrow \pm \infty$, respectively, and they have the same asymptotics at $\pm \infty$ as the solutions to the equation

$$
-\lambda^{2} u(x)=H_{0} u(x)
$$

where $H_{0}=-\partial_{x}^{2}$, which agrees with $H$ on $\mathbb{R} \backslash[-1,1]$. We call $J_{+}$and $J_{-}$the Jost solution to (29) and define the Evans function to be the Wronskian of $J_{+}$and $J_{-}$: 


$$
E(\lambda)=W\left(J_{+}, J_{-}\right)(x, \lambda)=J_{+}(x, \lambda) \partial_{x} J_{-}(x, \lambda)-J_{-}(x, \lambda) \partial_{x} J_{+}(x, \lambda),
$$

where the right-hand side depends only on $\lambda$. Vanishing of $E$ at some particular $\lambda \in \mathbb{C}, \operatorname{Re}(\lambda)<0$ shows that the Jost solutions $J_{+}$and $J_{-}$are linearly dependent, and there is $c \in \mathbb{C} \backslash\{0\}$ such that

$$
\phi(x)= \begin{cases}J_{+}(x, \lambda), & x \geq 0 \\ c J_{-}(x, \lambda), & x<0\end{cases}
$$

is $C^{1}$ and thus is an eigenfunction corresponding to an eigenvalue $\lambda^{2}$ of $H$.

The construction for the one-dimensional Soler model is done by decomposing $L^{2}\left(\mathbb{R}, \mathbb{C}^{4}\right)$ into two invariant subspaces for the operator $\mathscr{A}_{\omega}$ introduced in (9): the "even" subspace, with even first and third components and with odd second and fourth components, and the "odd" subspace, with odd first and third components and with even second and fourth components; the direct sum of the "even" and "odd" subspaces coincides with $L^{2}\left(\mathbb{R}, \mathbb{C}^{4}\right)$. The Evans function corresponding to the "even" subspace is defined by

$$
E_{\text {even }}(\lambda)=\operatorname{det}\left(R_{1}, R_{3}, J_{1}, J_{2}\right),
$$

where $R_{j}(x), 1 \leq j \leq 4$, are the solutions to the equation $\lambda R=\mathscr{A}_{\omega} R$ with the initial data

$$
\left.R_{j}\right|_{x=0}=\mathbf{e}_{j}, \quad 1 \leq j \leq 4,
$$

where $\mathbf{e}_{j}, 1 \leq j \leq 4$, is the standard basis in $\mathbb{C}^{4} . J_{1}$ and $J_{2}$ are the Jost solution of $\mathscr{A}_{\omega}$, which are defined as the solutions to $\lambda \Psi=\mathscr{A}_{\omega} \Psi$ with the same asymptotics at $+\infty$ as the solutions to $\lambda \Psi=\left(\mathbf{D}_{m}-\omega\right) \Psi$ which decay as $x \rightarrow+\infty$, where

$$
\mathbf{D}_{m}=\left[\begin{array}{cc}
D_{m} & 0 \\
0 & D_{m}
\end{array}\right], \quad D_{m}=\left[\begin{array}{cc}
m & \partial_{x} \\
-\partial_{x} & -m
\end{array}\right]=-i\left(-\sigma_{2}\right) \partial_{x}+m \sigma_{3}
$$

The Evans function corresponding to the "odd" subspace is constructed by using in (30) functions $R_{2}$ and $R_{4}$ instead of $R_{1}$ and $R_{3}$. We note that, by Liouville's formula, the right-hand side in (30) does not depend on $x$.

Fig. 9 shows the zeros of the Evans function which are plotted alongside with the essential spectrum for the linearization at the solitary waves in the 1D Soler model.

Later, in [46], it was observed that the linearized operator admits invariant subspaces which correspond to spinorial spherical harmonics. This allows one to factorize the operator, essentially reducing the consideration to a one-dimensional setting, and to perform a complete numerical analysis of the linearized stability in the nonlinear Dirac equation in two spatial dimensions and give partial results in three dimensions, basing our approach on both the Evans function technique and the linear stability analysis using spectral methods.

For the two-dimensional Soler model, we can use the same process as the onedimensional case to construct the Evans function. Recall (see (17)) that $\mathscr{A}_{\omega}$ acts invariantly on $\mathscr{X}_{q}$ for each $q \in \mathbb{Z}$ and $\mathscr{A}_{\omega, q}=\left.\mathscr{A}_{\omega}\right|_{\mathscr{X}_{q}}$. We consider the case $S=0$. 


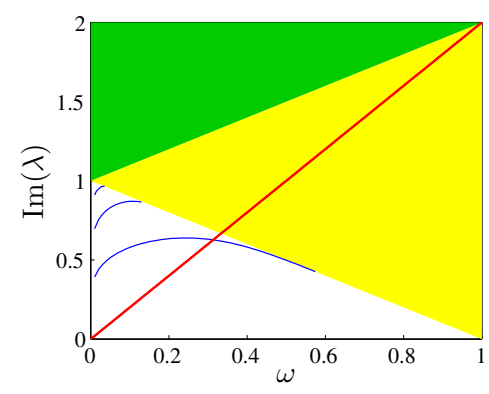

Fig. 9 Eigenvalues corresponding to zeros of the Evans function in the upper half of the spectral gap as a function of $\omega$. Yellow area represents the part of the continuous spectrum that corresponds to $i L_{ \pm}(\omega)$, while the green area represents the (doubly-covered) part of the continuous spectrum corresponding to both $i L_{ \pm}(\omega)$ and $-i L_{ \pm}(\omega)$. The eigenvalues $\lambda=2 \omega i$ (red straight line) are embedded into the essential spectrum for $\omega>m / 3$.

The Evans function for each $\mathscr{A}_{\omega, q}$ is defined by

$$
E_{q}(\lambda)=\operatorname{det}\left(R_{q}^{+}, R_{q}^{-}, Y_{1}, Y_{2}\right) .
$$

Here $R_{q}^{+}$and $R_{q}^{-}$are linearly independent solutions to the equation $\lambda R=\mathscr{A}_{\omega, q} R$ with the following linearly independent initial data at $r=0$

$$
\left[\begin{array}{c}
-\lambda-\left(\omega+f_{0}\right) \frac{i q}{|q|} \\
0 \\
i|q| \\
q
\end{array}\right] \text { and }\left[\begin{array}{c}
0 \\
-i \lambda \frac{q}{|q|}+\omega+f_{0} \\
|q| \\
i q
\end{array}\right]
$$

where $f_{0}=m-g\left(u^{2}(0)-v^{2}(0)\right)$. The Jost solutions $Y_{1}$ and $Y_{2}$ of $\mathscr{A}_{\omega, q}$ are defined as the solution to $\lambda Y=\mathscr{A}_{\omega, q} Y$ with the same asymptotics at $+\infty$ as the solutions to $\lambda Y=\mathbf{D}_{q} Y$ where

$$
\mathbf{D}_{q}=\left[\begin{array}{cc}
-\sigma_{1} \frac{i q}{r} & D_{m}-\omega I_{2} \\
-D_{m}+\omega I_{2} & -\sigma_{1} \frac{i q}{r}
\end{array}\right], \quad q \in \mathbb{Z} .
$$

\subsection{Bogoliubov-de Gennes analysis: The one-dimensional case}

Let us recall from the analysis shown in Section 4 that near the non-relativistic limit $(\omega \lesssim m)$, the stability of solitary waves formally agrees with the VakhitovKolokolov stability criterion $\partial_{\omega} Q\left(\phi_{\omega}\right)<0$ [151]. In particular, there is no positive eigenvalue emerging from $\lambda=0$ for $\omega \lesssim m$ as long as $k \leq 2$ (and, consequently, the solitary waves are spectrally stable), while in the case $k>2$ there is a pair of 
(a positive and a negative) eigenvalues which result in linear instability. As it turns out, in the one-dimensional case, the Vakhitov-Kolokolov stability criterion agrees with the observed stability of solitary waves not only in the nonrelativistic limit, but for all frequencies $\omega \in(0, m)$, as our numerical calculations show below. Evans function analysis presented above also shows that solitary waves do not present oscillatory instabilities (i.e. there are no complex $\lambda$ 's with nonzero real part) in the $1 \mathrm{D}$ case; the instability could only develop when eigenvalues collide and bifurcate from the origin. Additionally, for any $k$, the existence of an eigenvalue $\lambda= \pm 2 \omega i$ is a consequence of the $\mathbf{S U}(1,1)$-invariance of the Soler model [49, 72]. This mode, which does not give rise to any instability, is embedded into the essential spectrum for $\omega \in(m / 3, m)$ (see Fig.10).

Let us mention that it was shown in $[111,140]$ that attempts to apply Derrick's argument [53] to stability of solitary waves in the context of the nonlinear Dirac equation $[20,145]$ - in particular, the so-called Bogolubsky criterion - do not seem to work. This is not particularly surprising, given that Derrick's empirical argument, based on singling out one family of perturbations of a solitary wave and checking whether the solitary wave corresponds to the energy minimum on this curve, was introduced in the context of the second order systems, appealing to our Newtonianworld intuition. Apparently, this approach does not necessarily work in the context of the first order systems, such as the Dirac equation.

Another surprising result was explored by some of the present authors in [44]. It corresponds to the $\mathrm{BdG}$ analysis using finite difference discretization of the $1 \mathrm{D}$ Soler model; that is, the spatial derivatives $\partial_{x} f(x)$ in (7) are substituted by the central difference $\left(f_{n+1}-f_{n-1}\right) /(2 h)$. This method is tantamount to using the collocation points of (23) and (25) with $N$ collocation points, a domain $x \in[-L, L]$ and $h=$ $2 L / N$. Fig. 10 shows the stability eigenvalues for $k=1$ and $L=80$ while the spacing $h=0.1$. We do not consider here instabilities that disappear in the infinite domain, continuum limit .

In the case of small $\omega$, the solitary waves are identified as unstable. The "size" of the instability decreases as the frequency is increased. This is because of a mode colliding with the essential spectrum around $\omega \approx 0.037$ and leading to the formation of eigenfrequency quartets. For $\omega \approx 0.632$, the stability of the solitary wave is briefly restored, only to be lost again at $\omega \approx 0.634$. Subsequently, "bubbles" of instability arise (with decreasing amplitude as $\omega$ is increased). To identify the relevant trend, we have examined the cases of different length for $L=40,100$ and 150 . As $L$ increases, so does the number of bubbles, while the width decreases, with their envelope tending to zero when $\omega$ approaches 1 , in a way reminiscent of the corresponding scenario for dark solitons in the discrete nonlinear Schrödinger equation (DNLS) case [86]. From this trend, it is not straightforward to infer whether the unstable solution becomes stabilized at a critical $\omega$ or whether it is asymptotically approaching the stable NLS limit of $\omega \rightarrow 1$.

In order to find out a strategy which assures a spectral accuracy of BdG stability analysis which is also correlated to the Evans' function analysis, we used spectral collocation methods in [43]. We utilized two case examples of such methods therein: the Fourier Spectral Collocation Method, which implicitly enforces periodic bound- 

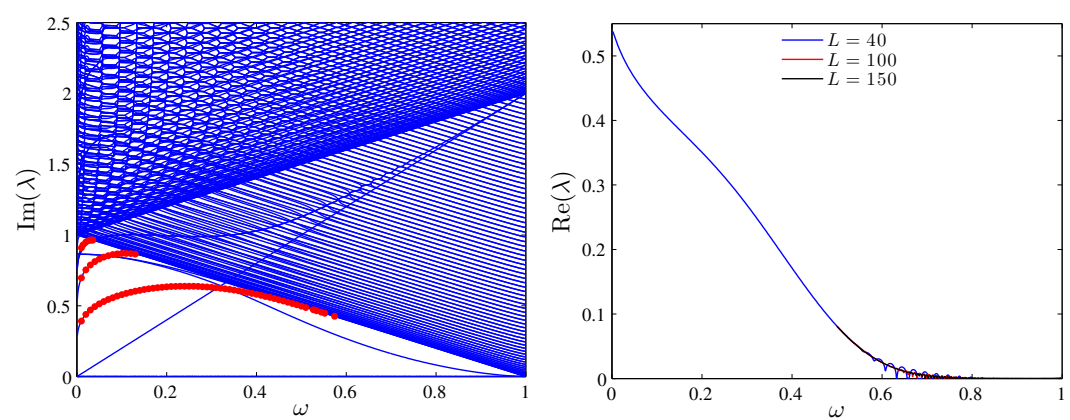

Fig. 10 Spectrum of the stability matrix (10) for solitary waves in domain $[-L, L]$ with $L=40$ obtained using finite differences with $N=800$ grid points in the cubic $(k=1)$ case. For the sake of simplicity, only the positive real and imaginary parts of the eigenvalues are shown. Dots correspond to Evans function predictions. Right panel displays only the maximum values of $\operatorname{Re}(\lambda)$ (i.e. the growth rates) and includes the values for $L=40, L=100$, and $L=150$.

ary conditions, and the Chebyshev Spectral Collocation Method, which enforces (homogeneous) Dirichlet boundary conditions (see Subsection 3.2). The advantage of the Finite Difference Method with respect to the other ones concerns the fact that the resulting stability matrix is sparse. In the computations performed in that work and that will be presented below, $N=800$ collocation points were taken in a domain $[-L, L]$, with a discretization parameter $h=1 /(2 L)$; this value coincides with the distance between grid points in the Fourier collocation and finite difference methods, but not in the Chebyshev collocation as the grid points are not equidistant. Increasing the node numbers to $N=1200$ does not seem to qualitatively improve the findings.

In Fig. 11 we examine the dependence of the imaginary part of the eigenvalues $\lambda$ with respect to the frequency $\omega$ of the solution for both spectral methods in the cubic case of $k=1$. In addition to the $\lambda= \pm 2 \omega i$ mode, the different methods have additional modes which can be compared also with the Evans function analysis outcome of Fig. 9. We thus find that the comparison of the Fourier spectral collocation method with the Evans function analysis (Fig. 9) seems qualitatively (and even quantitatively) to yield very good agreement with the exception of a mode that seems to initially grow steeply (for small $\omega$ ) and subsequently to slowly asymptote to the band edge (as $\omega$ increases). This mode is shown in the right panel of Fig. 12, while the left panel of the figure illustrates a prototypical example of the Fourier spectral collocation method spectrum for $\omega=0.1$. From the above panel, we can immediately infer that this mode is, in fact, spurious and an outcome of the discretization as it carries a staggered profile that cannot be supported in the continuum limit. In the left panel of the same figure, we can see the existence of additional spurious modes forming bubbles of complex eigenvalues. However, the fact that these bubbles are occurring at the eigenvalues of the continuous spectrum assures us that these are spurious instabilities due to the finite size of the domain and ones which disappear 

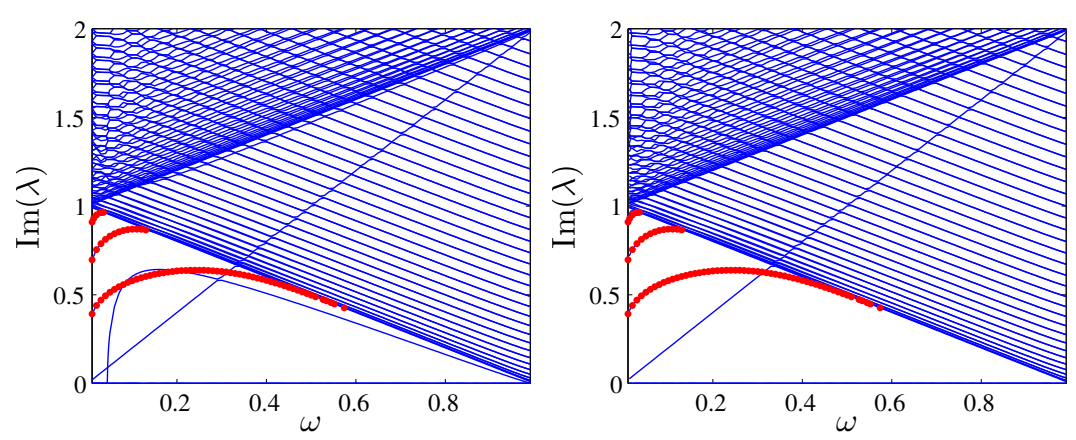

Fig. 11 Imaginary part of the spectrum of the stability matrix (10) for solitary waves in domain $[-L, L]$ with $L=40$ obtained using Fourier (left) and Chebyshev (right) spectral collocation method with $N=800$ grid points in the cubic $(k=1)$ case. For the sake of simplicity, only the positive imaginary parts of the eigenvalues are shown. Dots correspond to Evans function predictions.
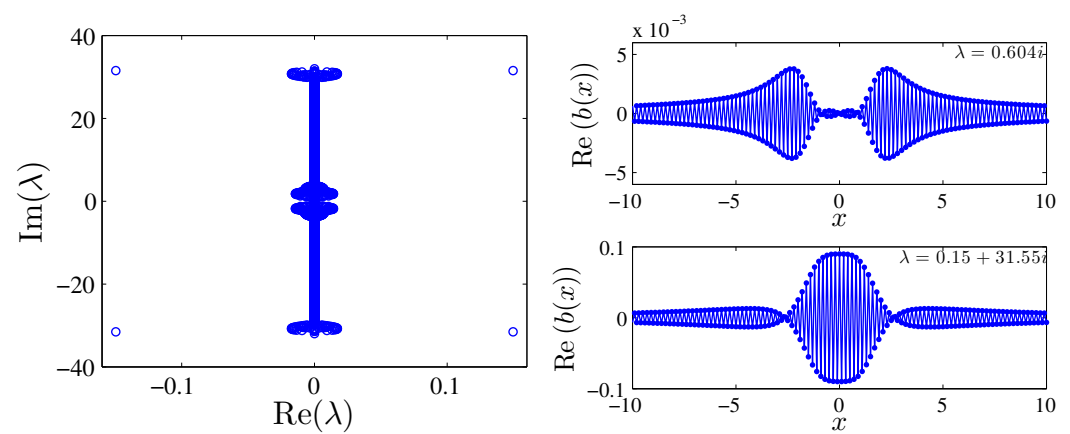

Fig. 12 Spectral plane of a solitary wave with $\omega=0.1$ (cubic case, $L=40$, and $N=800$ ) obtained using the Fourier spectral collocation method (left panel). The typical profile of two modes corresponding to spurious eigenvalues is depicted in the right panel. In particular, we have included the mode with $\operatorname{Re}(\lambda)=0$ which does not arise in the Evans' function analysis of Subsection 5.1 together with the largest real part eigenvalue, which is also spurious.

in the $L \rightarrow \infty, h \rightarrow 0$ limit. This is confirmed by Fig. 13 which shows that as we decrease $h$ (and increase the number of lattice sites, approaching the continuum limit for a given domain size) the growth rate of such spuriously unstable eigenmodes accordingly decreases.

Remarkably, the finite difference spectrum of Fig. 10 is the one that seems most "distant" from the findings of the Evans function method. While all four of the internal modes of the latter spectrum seem to be captured by the finite difference method, three additional modes create a nontrivial disparity. Two of them are in fact "benign" and maintain an eigenvalue below the band edge of the continuous spectrum for all values of $\omega \in(0, m)$. However, as explained in [44], we also observe the existence of an eigenmode embedded in the essential spectrum. Unfortunately, this mode is 


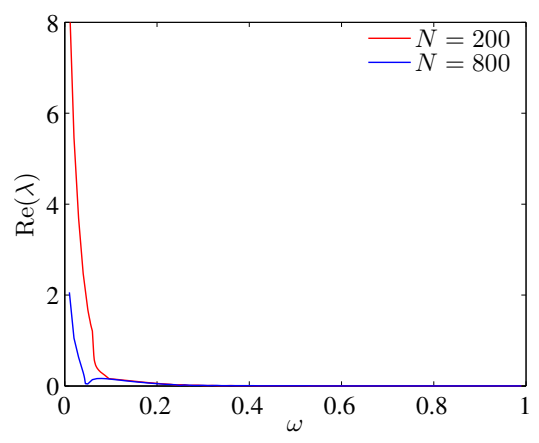

Fig. 13 Growth rates (i.e. maximum of the real part of the eigenvalues) for a solitary wave with $L=40$ in the cubic case using the Fourier spectral collocation method. The number of grid points is either $N=800$ or $N=200$.
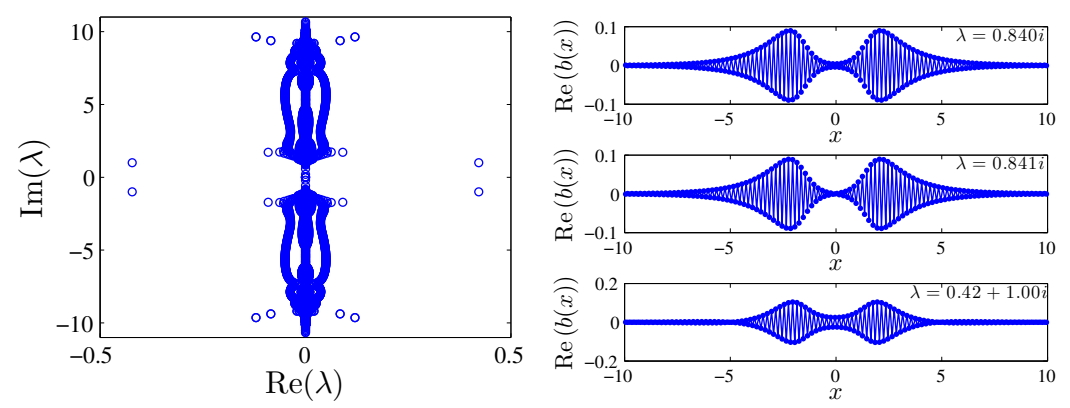

Fig. 14 Spectral plane of a solitary wave with $\omega=0.1, L=40$ and $N=800$ in the cubic case, using finite difference discretization (left panel). The typical profile of three modes corresponding to spurious eigenvalues is depicted in the right panel. In particular, we have included the two modes with $\operatorname{Re}(\lambda)=0$ which do not arise in the Evans function analysis together with the embedded spurious mode.

accompanied by a real part in the corresponding eigenvalue and hence gives rise to a spurious instability. Fig. 14 presents a graph analogous to Fig. 12 but for the finite difference method. The undesirable unstable mode, as well as additional spurious modes are explicitly indicated through the eigenvector profiles of the right panel.

The scenario of the Chebyshev spectral collocation method bears advantages and disadvantages in its own right. Although it gives an accurate result for the imaginary part of the eigenvalues, their real part grows for large $\operatorname{Im}(\lambda)$, as is also shown in Fig. 15. Additionally, as indicated in [28], approximately half of the values of the spectrum are spurious within the Chebyshev collocation methods, so they should be excluded from consideration. Furthermore, one can observe that in this case as well, spurious instability bubbles arise (see the right panel of Fig. 15), yet we have checked that these disappear in the continuum limit of $h \rightarrow 0$. 

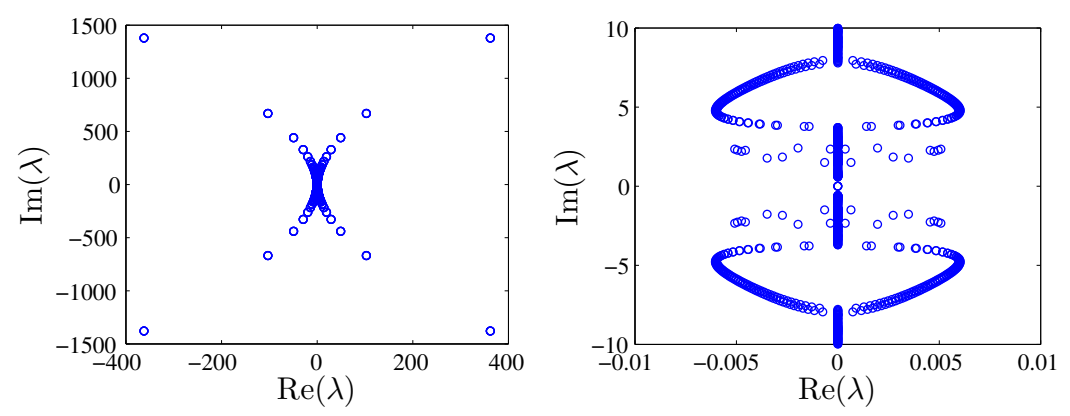

Fig. 15 Spectral plane of a solitary wave with $\omega=0.4, L=40$, and $N=800$ in the cubic case, using the Chebyshev spectral collocation method. The right panel is a zoom of that on the left, illustrating the weak, spurious instabilities (which disappear as the continuum limit is approached).

As a final aspect of the spectral considerations that we provide herein, we have examined the instability that arises e.g. from the Chebyshev spectral collocation method for larger values of $k$. Recall that the Chebyshev spectral collocation method predicts (at least as regards the point spectrum out of the non-embedded spectrum) that there is no instability for any $\omega$ in the case of $k=1$, in agreement with the Evans function analysis and [117]. The method identifies an instability for such point spectrum eigenvalues only for $k>2$. The relevant instability predicted numerically in the $k-\omega$ plane is illustrated in Fig. 16 . We note that this instability is precisely captured by the Vakhitov-Kolokolov criterion, i.e. it precisely corresponds to the condition $\partial_{\omega} Q(\omega)=0$, in agreement with [16]. Hence, by analogy with the nonrelativistic limit $\omega \rightarrow m=1$, we expect this to be an instability associated with the collapse of the latter model (however, we will observe a key dynamical difference, in comparison to the NLS, in Section 6). Nevertheless, it is relevant to point out here that the NLD, contrary to the NLS, does not exhibit an instability for all $\omega$ when $k>2$. The instability is instead limited to $\omega>\omega_{c}(k)$, as characterized by the curve of Fig. 16. Hence, it can be inferred that the instability is mitigated by the relativistic limit of the NLD and only occurs in an interval of frequency values including the non-relativistic limit $\omega \rightarrow m=1$, yet not encompassing the full range of available frequencies in the relativistic case.

\subsection{Bogoliubov-de Gennes analysis: The two- and three- dimensional cases}

From the experience acquired with the study of the stability of solitary waves in one spatial dimension, it is clear that a Chebyshev spectral collocation method must be followed in order to analyze the stability in higher-dimensional solitary waves. 


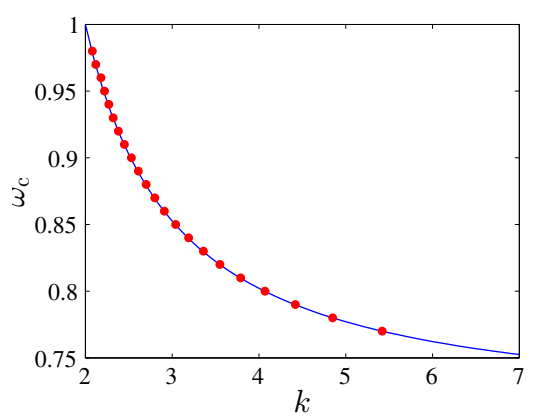

Fig. 16 Exponential bifurcation loci in the $\omega_{c}-k$ plane for the 1D Soler model. The solitary waves under the curve are linearly (spectrally) stable, while the ones above the curve are linearly unstable. Full line corresponds to the application of the Vakhitov-Kolokolov criterion (i.e. points for which $\partial_{\omega} Q(\omega)=0$ ), whereas the dots correspond to the stability calculations.

This is the approach followed in the present section, which summarizes the results of [46].

Let us remember that the spectrum of $\mathscr{A}_{\omega}$ is the union of spectra of the onedimensional spectral problems (17): $\sigma\left(\mathscr{A}_{\omega}\right)=\cup_{q \in \mathbb{Z}} \sigma\left(\mathscr{A}_{\omega, q}\right)$. In our numerics we have analyzed values of $q \in[-6,6]$, although the main phenomenology is captured by $|q| \leq 4$ and those are the values shown in the next figures for the sake of better visualization.

We start by considering the stability of We first examine $S=0$ structures in the case of cubic nonlinearity. In Fig. 17, we can see in the top panels how the real and imaginary parts of the eigenvalues depend on $\omega$. Based on this we can make some observations. Contrary to the case of the 2D NLS where the zero eigenvalues are degenerate [147], in the present NLD setting this degeneracy is lifted. As the frequency decreases, the pair of $q=0$ eigenvalues depart from the origin (where they are at $\omega=1$ ). Since these eigenvalues become marginally stable, the mechanism of charge-critical NLS self-similar blowup [110] is no longer "available". Moreover, the translation and gauge symmetry lead to two pairs of eigenvalues of eigenvalues at the origin, for both fundamental and excited solutions. An additional $(\mathbf{S U}(1,1))$ symmetry is responsible for the presence of eigenvalues $\lambda= \pm 2 \omega i$. It is relevant to note that as $\omega$ is decreased for $\omega<0.121$, Hamiltonian-Hopf bifurcations for $|q|=2$ lead to a complex eigenvalue quartet, with an additional one arising for $|q|=3$ at $\omega=0.0885$ and so on.

On the other hand, vortex solutions with $S>0$ were found to be generically unstable due to quartets of complex eigenvalues. This was true not only for $S=1$ solutions, but also for cases with $S \geq 2$ that we do not analyze further. Notice that the eigenvalues $\lambda= \pm 2 \omega i$ generally correspond to the particular mode with $q=$ $\mp(2 S+1)$.

Importantly, the quintic $(k=2)$ NLD model was also found to possess stable intervals in two dimensions. Here, the NLS limit is itself unstable and in fact the relevant instability emerges (for the $q=0$ perturbations) already for $\omega>0.890$. In 

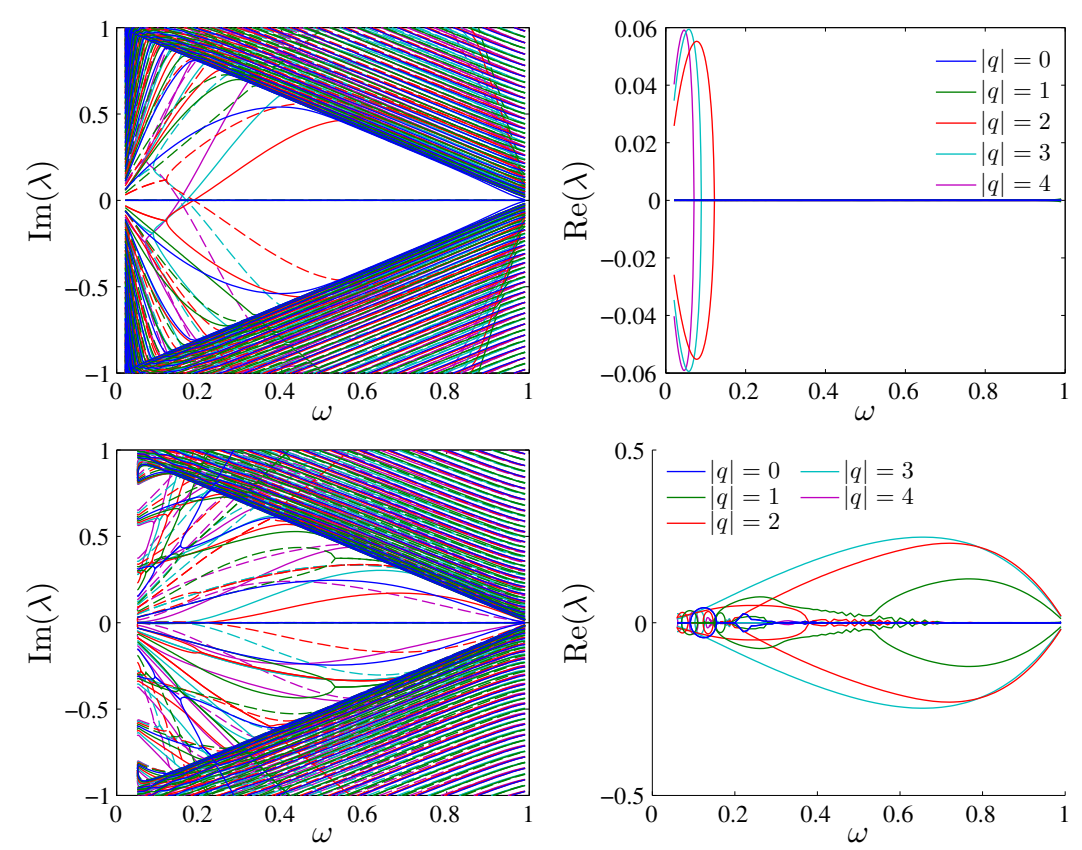

Fig. 17 Dependence of the (left) imaginary and (right) real part of the eigenvalues with respect to the frequency $\omega$ of solitary waves in the 2D Soler model with cubic $(k=1)$ nonlinearity. Top (respectively, bottom) panels correspond to $S=0$ solitary waves ( $S=1$ vortices). For the sake of clarity, we only included the values $|q| \leq 4$. Full (dashed) lines in left panels represent the eigenvalues for $q \geq 0(q<0)$. The correspondence between colors and $|q|$ is indicated in the legend of right panels.

the small $\omega$ realm, the instability sets in (via Hamiltonian Hopf bifurcations again) for $\omega<0.312$.

Perhaps even more remarkably, Fig. 19 shows that the radial perturbations do not destabilize NLD solitary waves even in the case of 3D Soler models for suitable frequency intervals (entirely contrary to what is the case for the non-relativistic NLS limit). More specifically, the stability to radial perturbations arises below some dimension-dependent critical value $\omega_{c}=\omega_{c}(n, k)$, with $n$ being the number of spatial dimensions.

Fig. 20 shows those critical frequencies as a function of the nonlinearity parameter $k$ for $n=2$ and $n=3$. For $\omega \in\left(\omega_{c}, 1\right)$, the NLD solitary waves are linearly unstable. Below $\omega_{c}$ the linear instability disappears. In the particular case of cubic $(k=1)$ 3D Soler model, we have that $\omega_{c} \approx 0.936$. This value was identified by Soler in his original paper [143] as the parametric value of the occurrence of the energy and charge minimum. 

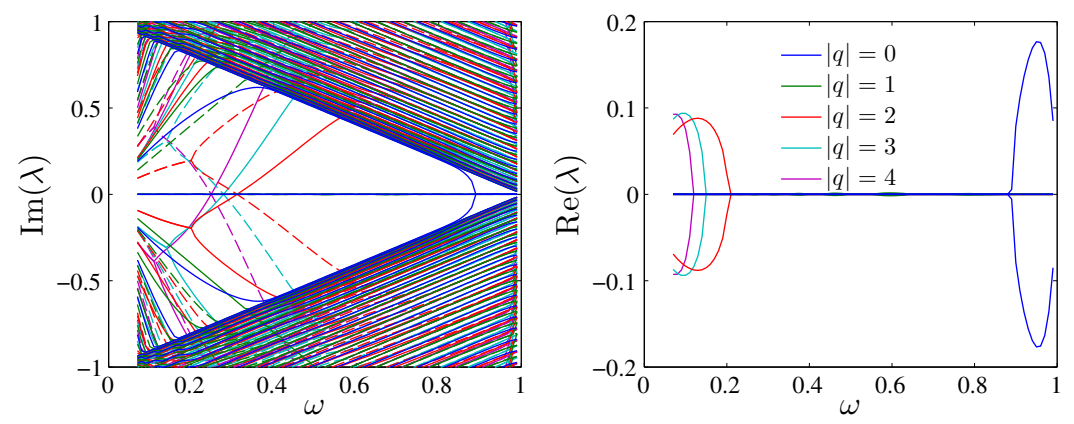

Fig. 18 Dependence of the (left) imaginary and (right) real part of the eigenvalues with respect to the frequency $\omega$ of $S=0$ solitary waves in the 2D Soler model with quintic $(k=2)$ nonlinearity. For the sake of clarity, we only included the values $|q| \leq 4$. Full (dashed) lines in the left panel represent the eigenvalues for $q \geq 0(q<0)$. The correspondence between colors and $|q|$ is indicated in the legend of the right panel.
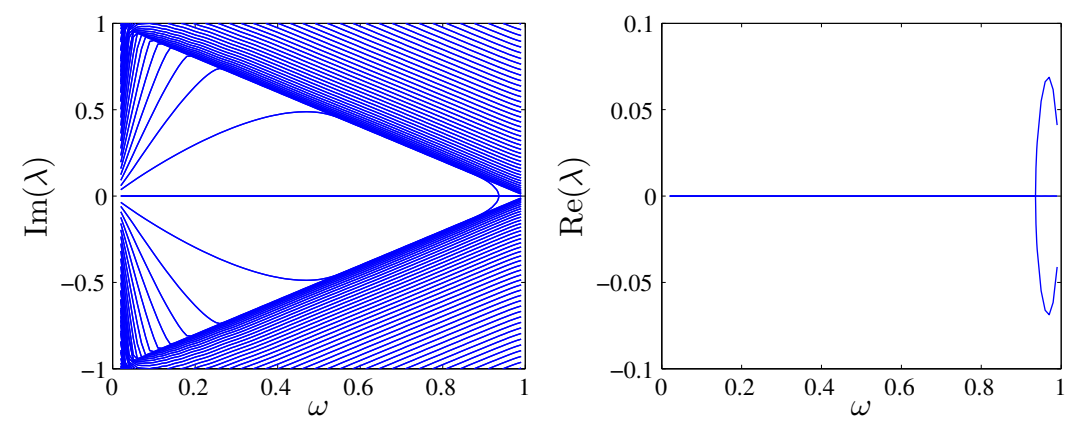

Fig. 19 Dependence with respect to $\omega$ of the (left) imaginary and (right) real part of the eigenvalues of the one-dimensional invariant $(q=0)$ subspace of $S=0$ solitary waves in the 3D Soler model with cubic $(k=1)$ nonlinearity.

\section{Dynamics}

Once the stability properties of solitary waves and vortices of the Soler model have been elucidated, it is now natural to turn our attention towards the observation of their dynamical properties. In the one-dimensional case, we will analyze some integration schemes in order to observe their suitability for simulation of solitary waves in nonlinear Dirac equations. In addition, the dynamics of unstable solutions in equations with high-order instabilities (i.e. $k>1$ ) will be shown. Finally, the dynamics of unstable solitary waves and vortices for the 2D Soler model will be considered. 


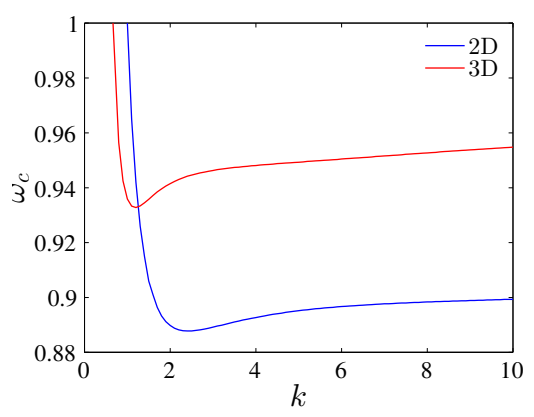

Fig. 20 Critical frequency for radially-symmetric $(q=0)$ exponential bifurcations in the 2D and 3D Soler model, as a function of the exponent $k$. For $\left(\omega_{c}, 1\right)$, the $S=0$ solitary waves are linearly unstable. For $k \leq 2 / n$, with $n$ being the system dimension, there is no linear instability for $\omega \lesssim 1$, according to the Vakhitov-Kolokolov criterion (see Section 4).

\subsection{One-dimensional solutions}

This subsection is divided into two parts. In the first one, we will show the evolution of stable solitary waves within several numerical integrators in the cubic $(k=1)$ Soler model. The second part deals with the evolution of unstable solitary waves with $k>1$. Most of the results presented herein are taken from [43].

\subsubsection{Stable solutions}

We turn here our attention to the implications of spectral collocation methods to the nonlinear dynamical evolution problem. We focus on the case of $k=1$. Given the large (yet spurious) growth rate of the modes emerging from the Chebyshev spectral collocation method and the spurious point spectrum instability of the finite difference method, for our dynamical considerations, we will focus our attention to the Fourier spectral collocation method results. As discussed in Subsection 5.2, in that method too, there exist spurious modes which, as expected, are found to affect the corresponding dynamics. As a dynamical outcome of these modes, the solitary waves are found to be destroyed after a suitably long evolution time, although the time for this feature is controllably longer in comparison to the one observed in [140]. This, in turn, suggests the expected stability of the solitary wave solutions, in accordance with what was proposed in Section 5.

As a prototypical diagnostic of the dynamical stability of solitary waves in a finite domain $[-L, L]$, we have monitored the $L^{2}$-error in a similar fashion as in [140]:

$$
\varepsilon_{2}(t)=\left(\int|\rho(t, x)-\rho(0, x)|^{2} d x\right)^{1 / 2}
$$

with $\rho=\psi^{*} \psi$ being the charge density. 
A first approach to the dynamics problem is accomplished by choosing a fixedstep 4th order Runge-Kutta method. We observe that the lifetime is longer when the frequency $\omega$ is fixed and the domain length $L$ is increased. This is associated with the decrease of the size of spurious instability bubbles, as we approach the infinite domain limit. A similar decrease of the growth rate is observed for a given $L$, when the discretization spacing $h$ is decreased (i.e., as the continuum limit is approached), in accordance with the spectral picture of Fig. 13. In addition, if $L$ is fixed, the lifetime is longer when $\omega$ is increased. This is summarized in Fig. 21. This is, of course, in consonance with earlier observations such as those of [140], however, our ability to expand upon the lifetimes as the domain and discretization parameters are suitably tuned suggests that in the infinite domain, continuum limit such instabilities could be made to disappear upon suitable selection of the numerical scheme. As a final comment, we note that the growth rates observed in Fig. 21 are consonant with the maximal (yet spurious) instability growth identified in Fig. 13. This is yet another indication that this growth featured in the time dynamics is a spurious byproduct of the discretization scheme, rather than a true feature of the corresponding continuum problem.
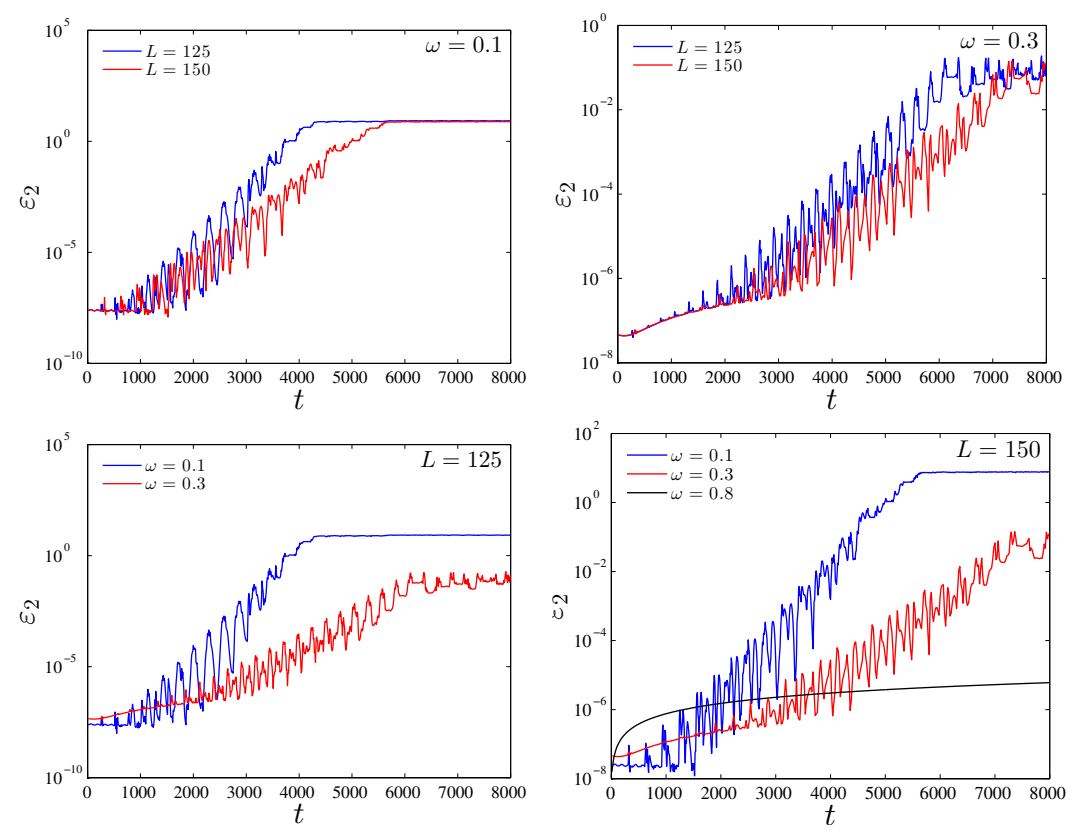

Fig. 21 Stable solitary waves simulations in cubic 1D Soler model using a 4th-order Runge-Kutta integrator with a Fourier spectral collocation method. The norm error is compared for different domain sizes and frequencies. In every case, the time step of the integrator is $\Delta t=0.05$. 
Table 1 Comparison between the critical times for which $\varepsilon_{2}>10^{-3}$ using the Fourier Spectral Collocation method with a 4th-order Runge-Kutta integrator $\left(t_{1}\right)$ and the Operator Splitting Method of [140] $\left(t_{2}\right)$.

\begin{tabular}{|c|c|c|c|c|}
\hline \multirow[b]{2}{*}{$L$} & \multicolumn{2}{|c|}{$\omega=0.1$} & \multicolumn{2}{|c|}{$\omega=0.5$} \\
\hline & $t_{1}$ & $t_{2}$ & $t_{1}$ & $t_{2}$ \\
\hline 50 & 1220 & 121 & 5620 & 6614 \\
\hline 75 & 1320 & 122 & 8480 & 8724 \\
\hline 100 & 1990 & 122 & 14660 & 9937 \\
\hline 125 & 2540 & 120 & 14660 & 11670 \\
\hline 150 & 3120 & 122 & 14660 & 13560 \\
\hline
\end{tabular}

In Table 1 we compare the critical time for which $\varepsilon_{2}>10^{-3}$ within the Fourier spectral collocation method and the corresponding time for the 4th order operator splitting algorithm used in [140] for which we have the frequencies $\omega=0.1$ and $\omega=$ 0.5 and different domain lengths $L$. As can be seen from the comparison, although in some cases (e.g. for $\omega=0.5$ and $L=50$ ) the observed destabilization may happen later for the scheme of [140], generally the Fourier spectral collocation method code explored herein allows to enhance the wave lifetime, in some cases by an order of magnitude. This can be further improved by tweaking parameters such as $h$ and the time spacing of the integrator $\Delta t$, as discussed above. Hence, our conclusion is that despite the artificial instabilities existing in the spectral picture and their dynamical manifestation, it is anticipated that the continuum, real line variant of the problem is spectrally stable for all $\omega \in(0, m)$ in the case of $k=1$.

A tweak to the problem could be, on the one hand, to use adaptive step-size integrators [80]. The case of 4th-5th order Dormand-Prince integrator [55] does not improve significantly the solitary wave lifetime. On the other hand, when using a 2nd-3rd order Runge-Kutta integrator supplemented by a TR-BDF2 scheme (i.e. a trapezoidal rule step as a first stage and a backward differentiation formula as a second stage) [139], many of the spurious eigenvalues can be damped out and the lifetimes are strongly enhanced.

\subsubsection{Unstable solutions for high-order nonlinearity}

Having observed that the solitary wave solutions of the problem with $k=1$ (and, in fact, with any $k<2$ ) are dynamically stable, we now turn our attention to the dynamics associated with the instability in the case $k>2$, for $\omega>\omega_{c}(k)$, as per Fig. 16. Figure 22 shows the evolution of an exponentially unstable solitary wave with $k=3$ and $\omega=0.9$. We can observe the existence of oscillations around a stable fixed point. This fixed point approximately corresponds to the solitary wave with frequency $\omega \approx 0.82$, for which the solution is spectrally stable. This is in stark contrast with the supercritical dynamics of the Nonlinear Schrödinger equation. There, 
the instability directly leads to collapse and an indefinite growth of the amplitude of the solution. On the contrary, in the case of the Soler model, for any value of $k$ for which the solution may become unstable, there exists (for the same $k$ ) an interval of spectrally stable states of the same type. Hence, the solution to the Soler model does not escape towards collapse but rather departs from the vicinity of the unstable fixed point solution and finds itself orbiting around a center, i.e., a stable solitary wave structure.
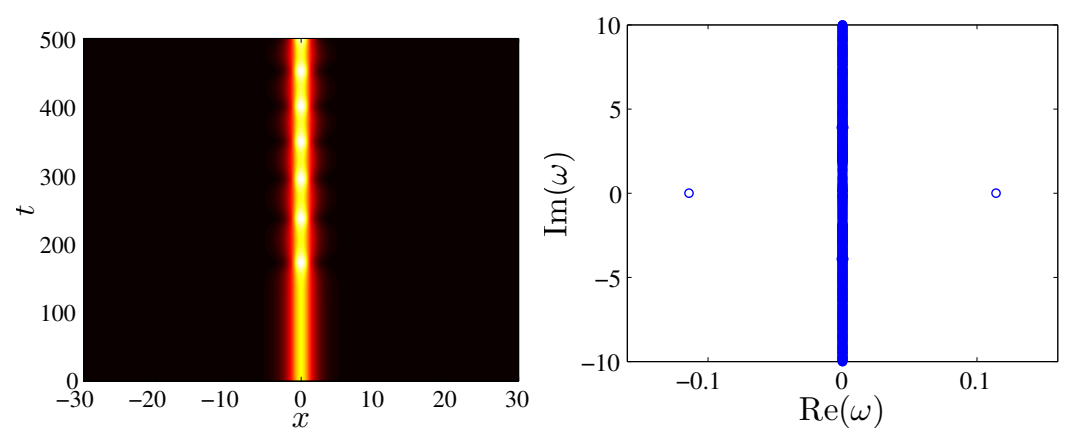

Fig. 22 (Left) Time evolution of a 1D solitary wave with nonlinearity exponent $k=3$ and frequency $\omega=3$. (Right) Spectral plane of the solitary wave whose evolution in traced in the left panel.

\subsection{Two-dimensional solutions}

This subsection reviews the results on the dynamics of 2D solitary waves and vortices shown in [46]. In order to simulate their dynamics, Chebyshev spectral methods and finite difference methods are not the most suitable ones, because of the presence of many spurious eigenvalues, and the dimensionality of the problem makes the TR-BDF2 schemes difficult to implement because of the high memory requirements. Thus, it seems that the optimal way to proceed is to use a Fourier spectral collocation method, which, as shown for the 1D problem, works fairly well as long as the frequency $\omega$ is not close to zero.

Consequently, periodic boundary conditions must be supplied to our problem. This is less straightforward when working in polar coordinates in the domain $(0, L) \times[0,2 \pi)$. For this reason, we opt to work with a purely $2 \mathrm{D}$ problem in rectangular coordinates in the domain $(-L, L] \times(-L, L]$. The simulations we show below have been performed with a Dormand-Prince numerical integrator using such a spectral collocation scheme with the aid of Fast Fourier Transforms (24).

A prototypical example of the evolution of the instability of the fundamental solitary waves for $k=1$ is shown in Fig. 23. As can be observed, the radial symmetry 

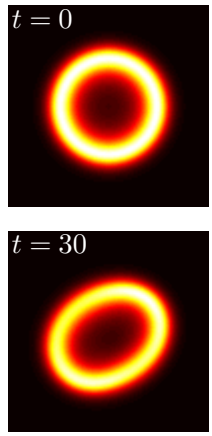
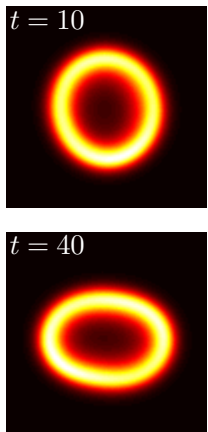
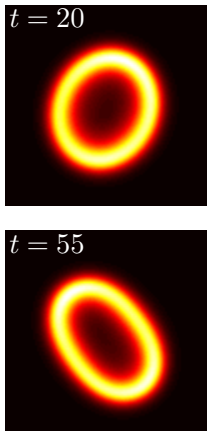
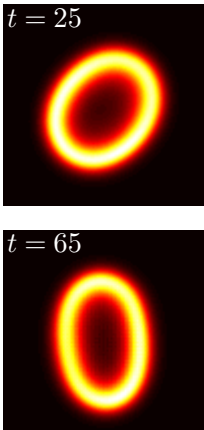

Fig. 23 Snapshots showing the evolution of the density of an unstable $S=0$ solitary wave with $\omega=0.12$ in the cubic $2 \mathrm{D}$ Soler model. The solitary wave which initially had a circular shape becomes elliptical and rotates around the center of the original solitary wave.
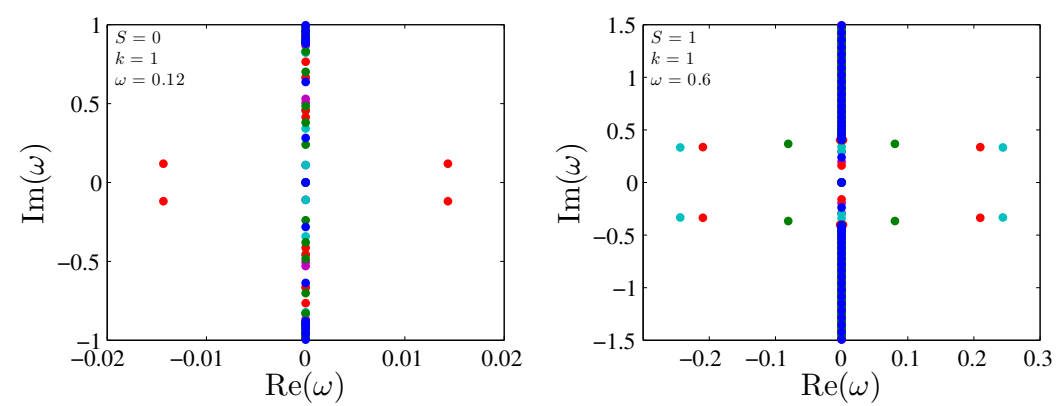

Fig. 24 Spectral planes of the unstable solitary waves whose dynamics are depicted in Figs. 23 and 25 (left and right panels, respectively). Each color represents a different value of $q$ as in Fig. 17.

in the density of $S=0$ solitary waves is spontaneously broken and, as a result, the coherent structures acquire an elliptical shape, rotating around the density center of the original coherent structure, as may be expected by the quadrupolar $(q=2)$ nature of the unstable mode. (see spectrum at the left panel of Fig. 24). The dynamical outcome of $S=1$ vortices for $k=1$ is shown in Fig. 25, whose instability (see spectral plane at right panel of Fig. 24) leads to the splitting into three smaller ones; in particular, the first spinor splits into fundamental structures, while the second one leads to the formation of vortices in a way preserving the total vorticity across the components. In the case of an $S=2$ vortex, five similar structures arise, again preserving the total vorticity (see Fig. 26).

As a final example, we showcase the effect of radial $k=2$ perturbations in the unstable case of $\omega>\omega_{c}$ (see Fig. 27). We can see that the relevant dynamics amounts to a breathing pattern, without featuring collapse. (similarly e.g. to Fig. 22. 

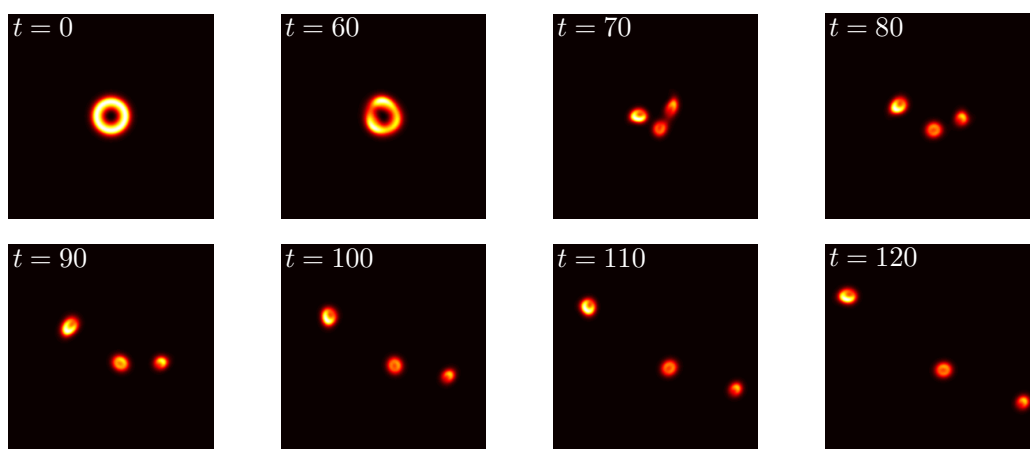

Fig. 25 Snapshots showing the evolution of the density of an unstable $S=1$ vortex with $\omega=0.6$ in the cubic 2D Soler model.
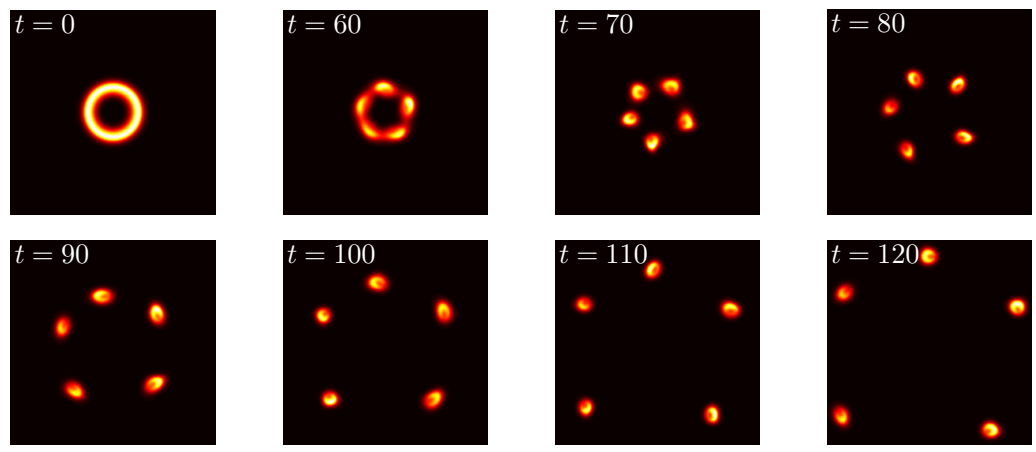

Fig. 26 Snapshots showing the evolution of the density of an unstable $S=2$ vortex with $\omega=0.6$ in the cubic 2D Soler model.

Fig. 27 Isosurface for the density of the $S=0$ solitary wave in the quintic $2 \mathrm{D}$ Soler model with $\omega=0.94$.

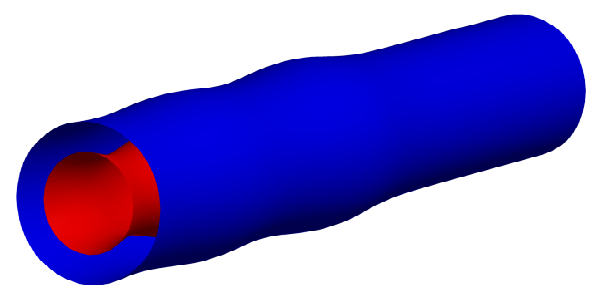




\section{Summary and outlook}

In the present work, we have discussed some of the principal properties of the nonlinear Dirac equation and its similarities, as well as differences, in comparison to its extensively studied cousin, namely the nonlinear Schrödinger equation. We have discussed nonlinear models that possess solitary wave solutions and vortices, and have placed particular emphasis on their spectral stability, also mentioning the orbital and asymptotic stability thereof and the corresponding issues that arise. We have seen that especially in higher dimensions the stability properties of solitary waves in the nonlinear Dirac equation can be fundamentally different from the NLS case, and may not feature collapse scenarios. Moreover, solitary waves may be spectrally stable for suitable parametric (i.e., frequency) regimes. For the threedimensional case, the stability properties are just starting to be explored (in suitable subspaces), yet this problem is extremely interesting, also due to its connections with the dynamics. In the context of the latter, we explored some of the delicate features that arise from different types of discretizations (finite-difference, Fourier and Chebyshev spectral schemes) and the implications for the evolutionary dynamics. Generally, we hope to have exposed some of the significant complications arising in dynamically propagating such a system, especially when trying to do so for long time scales.

From every perspective that we can think of, nonlinear Dirac systems pose significant challenges ahead of us. From the point of view of the mathematical analysis, understanding the spectral properties observed herein and their dynamical implications is already a formidable problem. Computing efficiently and systematically both the solutions and their linearization eigenvalues emerges as a significant and upcoming challenge. This is especially true in three spatial dimensions. Devising numerical schemes - possibly based on integrable (semi-discrete or genuinely discrete) variants of the model - could prove to be of paramount importance towards future robust computations of the dynamics. Finally, combining some of the cutting edge themes in nonlinear waves (such as for instance rogue waves [93]) with relevant scenarios involving Dirac-type nonlinear models opens another highly promising vein of research for future studies [52].

\section{Acknowledgments}

The research of Andrew Comech was carried out at the Institute for Information Transmission Problems of the Russian Academy of Sciences at the expense of the Russian Foundation for Sciences (project 14-50-00150). J.C.-M. thanks financial support from MAT2016-79866-R project (AEI/FEDER, UE). P.G.K. gratefully acknowledges the support of NSF-PHY-1602994, the Alexander von Humboldt Foundation, the Stavros Niarchos Foundation via the Greek Diaspora Fellowship Program, and the ERC under FP7, Marie Curie Actions, People, International Research 
Staff Exchange Scheme (IRSES-605096). This work was supported in part by the U.S. Department of Energy.

\section{References}

1. Ablowitz, M.J., Nixon, S.D., Zhu, Y.: Conical diffraction in honeycomb lattices. Phys. Rev. A 79, 053830 (2009)

2. Ablowitz, M.J., Prinari, B., Trubatch, A.D.: Discrete and continuous Nonlinear Schrödinger systems. Cambridge University Press, Cambridge (2004)

3. Ablowitz, M.J., Zhu, Y.: Evolution of Bloch-mode envelopes in two-dimensional generalized honeycomb lattices. Phys. Rev. A 82, 013840 (2010)

4. Achilleos, V., Frantzeskakis, D.J., Kevrekidis, P.G., Pelinovsky, D.E.: Matter-wave bright solitons in spin-orbit coupled Bose-Einstein condensates. Phys. Rev. Lett. 110, 264101 (2013)

5. Achilleos, V., Stockhofe, J., Kevrekidis, P.G., Frantzeskakis, D.J., Schmelcher, P.: Matterwave dark solitons and their excitation spectra in spin-orbit coupled Bose-Einstein condensates. Europhys. Lett. 103(2), 20002 (2013)

6. Alexander, J., Gardner, R., Jones, C.: A topological invariant arising in the stability analysis of traveling waves. J. Reine Angew. Math 410, 167-212 (1990)

7. Alvarez, A., Soler, M.: Energetic stability criterion for a nonlinear spinorial model. Phys. Rev. Lett. 50, 1230-1233 (1983)

8. Alvarez, A., Soler, M.: Stability of the minimum solitary wave of a nonlinear spinorial model. Phys. Rev. D 34, 644-645 (1986)

9. Barashenkov, I.V., Pelinovsky, D.E., Zemlyanaya, E.V.: Vibrations and oscillatory instabilities of gap solitons. Phys. Rev. Lett. 80, 5117-5120 (1998)

10. Bender, C.M.: Making sense of non-Hermitian Hamiltonians. Rep. Prog. Phys. 70, 947-1018 (2007)

11. Bender, C.M., Berntson, B.K., Parker, D., Samuel, E.: Observation of PT phase transition in a simple mechanical system. Am. J. Phys 81, 173-179 (2013)

12. Bender, C.M., Fring, A., Günther, U., Jones, H.: Special issue: Quantum physics with nonhermitian operators. J. Phys. A, Math Theor. 45(44), 020201 (2012)

13. Bender, C.M., Jones, H.F., Rivers, R.J.: Dual PT-symmetric quantum field theories. Phys. Lett. B 625, 333-340 (2005)

14. Berestycki, H., Lions, P.L.: Nonlinear scalar field equations. I. Existence of a ground state. Arch. Rational Mech. Anal. 82(4), 313-345 (1983)

15. Berkolaiko, G., Comech, A.: On spectral stability of solitary waves of nonlinear dirac equation in 1D. Math. Model. Nat. Phenom. 7, 13-31 (2012)

16. Berkolaiko, G., Comech, A., Sukhtayev, A.: Vakhitov-Kolokolov and energy vanishing conditions for linear instability of solitary waves in models of classical self-interacting spinor fields. Nonlinearity 28(3), 577-592 (2015)

17. Berthier, A., Georgescu, V.: On the point spectrum of Dirac operators. J. Funct. Anal. 71(2), 309-338 (1987)

18. Bjorken, J., Drell, S.: Relativistic quantum mechanics. McGraw-Hill, New York (1964)

19. Blanchard, P., Stubbe, J., Vázquez, L.: Stability of nonlinear spinor fields with application to the Gross-Neveu model. Phys. Rev. D 36, 2422-2428 (1987)

20. Bogolubsky, I.L.: On spinor soliton stability. Phys. Lett. A 73, 87-90 (1979)

21. Bournaveas, N.: Local existence for the Maxwell-Dirac equations in three space dimensions. Comm. Partial Differential Equations 21(5-6), 693-720 (1996)

22. Boussaïd, N.: Stable directions for small nonlinear Dirac standing waves. Comm. Math. Phys. 268(3), 757-817 (2006)

23. Boussaïd, N.: On the asymptotic stability of small nonlinear Dirac standing waves in a resonant case. SIAM J. Math. Anal. 40(4), 1621-1670 (2008) 
24. Boussaïd, N., Comech, A.: On spectral stability of the nonlinear Dirac equation. J. Funct. Anal. 271(6), 1462-1524 (2016)

25. Boussaïd, N., Comech, A.: Spectral stability of weakly relativistic solitary waves of the Dirac equation with the Soler-type nonlinearity (2016)

26. Boussaïd, N., Comech, A.: Spectral stability of weakly relativistic solitary waves of the Dirac equation with the Soler-type nonlinearity (2017). To appear

27. Boussaïd, N., Cuccagna, S.: On stability of standing waves of Nonlinear Dirac equations. Commun. Part. Diff. Eq. 37, 1001-1056 (2012)

28. Boyd, J.P.: Chebyshev and Fourier spectral methods, 2nd. edition edn. Dover, New York (2001)

29. Braun, O.M., Kivshar, Y.S.: The Frenkel-Kontorova Model. Springer Nature (2004)

30. Buslaev, V.S., Perel'man, G.S.: On the stability of solitary waves for nonlinear Schrödinger equations. In: Nonlinear evolution equations, Amer. Math. Soc. Transl. Ser. 2, vol. 164, pp. 75-98. Amer. Math. Soc., Providence, RI (1995)

31. Candy, T.: Global existence for an $L^{2}$ critical nonlinear Dirac equation in one dimension. Adv. Differential Equations 16(7-8), 643-666 (2011)

32. Cazenave, T., Lions, P.L.: Orbital stability of standing waves for some nonlinear Schrödinger equations. Comm. Math. Phys. 85(4), 549-561 (1982)

33. Cazenave, T., Vazquez, L.: Existence of localized solutions for a classical nonlinear Dirac field. Commun. Math. Phys. 105, 35-47 (1986)

34. Chugunova, M., Pelinovsky, D.: Block-diagonalization of the symmetric first-order coupledmode system. SIAM J. Appl. Dyn. Syst. 5(1), 66-83 (2006)

35. Coleman, S.: Quantum sine-Gordon equation as the massive Thirring model. Phys. Rev. D 11, 2088-2097 (1975)

36. Comech, A., Guan, M., Gustafson, S.: On linear instability of solitary waves for the nonlinear Dirac equation. Ann. I. H. Poincaré - AN 31, 639-654 (2014)

37. Comech, A., Phan, T.V., Stefanov, A.: Asymptotic stability of solitary waves in generalized Gross-Neveu model. Ann. Inst. H. Poincaré - AN 34, 157-196 (2017)

38. Comech, A., Stuart, D.: Small solitary waves in the Dirac-Maxwell system (2012). ArXiv: 1210.7261

39. Conduit, G.J.: Line of Dirac monopoles embedded in a Bose-Einstein condensate. Phys. Rev. A 86, 021605(R) (2012)

40. Contreras, A., Pelinovsky, D.E., Shimabukuro, Y.: $\mathrm{L}^{2}$ orbital stability of Dirac solitons in the massive Thirring model. Commun. Part. Diff. Eq. 41, 227-255 (2016)

41. Cooper, F., Khare, A., Mihaila, B., Saxena, A.: Solitary waves in the nonlinear Dirac equation with arbitrary nonlinearity. Phys. Rev. E 82, 036604 (2010)

42. Cuccagna, S., Tarulli, M.: On stabilization of small solutions in the nonlinear Dirac equation with a trapping potential. J. Math. Anal. Appl. 436(2), 1332-1368 (2016)

43. Cuevas-Maraver, J., Kevrekidis, P., Saxena, A., Cooper, F., Mertens, F.: Solitary waves in the nonlinear Dirac equation at the continuum limit: Stability and dynamics. In: Ordinary and Partial Differential Equations. Nova Science Publishers, New York (2015)

44. Cuevas-Maraver, J., Kevrekidis, P.G., Saxena, A.: Solitary waves in a discrete nonlinear Dirac equation. J. Phys. A: Math. Theor. 48, 055204 (2015)

45. Cuevas-Maraver, J., Kevrekidis, P.G., Saxena, A., Comech, A., Lan, R.: Stability of solitary waves and vortices in a 2D nonlinear Dirac model. Phys. Rev. Lett. 116, 214101 (2016)

46. Cuevas-Maraver, J., Kevrekidis, P.G., Saxena, A., Cooper, F., Khare, A., Comech, A., Bender, C.M.: Solitary waves of a PT-symmetric Nonlinear Dirac equation. IEEE J. Sel. Top. Quantum Electron. 22, 5000109 (2016)

47. Cuevas-Maraver, J., Kevrekidis, P.G., Williams, F. (eds.): The sine-Gordon Model and its Applications. Springer International Publishing (2014)

48. Dalibard, J., Gerbier, F., Juzeliunas, G., Öhberg, P.: Colloquium: Artificial gauge potentials for neutral atoms. Rev. Mod. Phys. 83, 1523-1543 (2011)

49. Darby, D., Ruijgrok, T.W.: A noncompact gauge group for the Dirac equation. Acta Phys. Polon. B 10, 959-973 (1979) 
50. Dauxois, T., Peyrard, M.: Physics of solitons. Cambridge University Press, Cambridge (2006)

51. De Wit, B., Smith, J.: Field Theory in Particle Physics. North Holland Physics Publishing, New York (1986)

52. Degasperis, A., Wabnitz, S., Aceves, A.: Bragg grating rogue wave. Phys. Lett. A 379, 10671070 (2015)

53. Derrick, G.H.: Comments on nonlinear wave equations as models for elementary particles. J. Math. Phys. 5, 1252-1254 (1964)

54. Dirac, P.: The quantum theory of the electron. I. Proc. R. Soc. Lond. A 117, 610-624 (1928)

55. Dormand, J.R., Prince, P.J.: A family of embedded runge-kutta formulae. J. Comp. Appl. Math. 6, 19-26 (1980)

56. Escobedo, M., Vega, L.: A semilinear Dirac equation in $H^{s}\left(\mathbf{R}^{3}\right)$ for $s>1$. SIAM J. Math. Anal. 28(2), 338-362 (1997)

57. Esteban, M.J., Georgiev, V., Séré, É.: Stationary solutions of the Maxwell-Dirac and the Klein-Gordon-Dirac equations. Calc. Var. Partial Differential Equations 4(3), 265-281 (1996)

58. Esteban, M.J., Lewin, M., Séré, É.: Variational methods in relativistic quantum mechanics. Bull. Amer. Math. Soc. (N.S.) 45(4), 535-593 (2008)

59. Esteban, M.J., Séré, E.: Stationary states of the nonlinear Dirac equation: A variational approach. Commun. Math. Phys. 171, 323-350 (1995)

60. Esteban, M.J., Séré, É.: Solutions of the Dirac-Fock equations for atoms and molecules. Comm. Math. Phys. 203(3), 499-530 (1999)

61. Esteban, M.J., Séré, E.: Nonrelativistic limit of the Dirac-Fock equations. Ann. Henri Poincaré 2(5), 941-961 (2001)

62. Esteban, M.J., Séré, É.: Dirac-Fock models for atoms and molecules and related topics. In: XIVth International Congress on Mathematical Physics, pp. 21-28. World Sci. Publ., Hackensack, NJ (2005)

63. Evans, J.: Nerve axon equations, I: Linear approximations. Indiana U. Math. J. 21, 877-955 (1972)

64. Evans, J.: Nerve axon equations, II: Stability at rest. Indiana U. Math. J. 22, 75-90 (1972)

65. Evans, J.: Nerve axon equations, III: Stability of the nerve impulse. Indiana U. Math. J. 22, 577-594 (1972)

66. Evans, J.: Nerve axon equations, IV: The stable and unstable impulse. Indiana U. Math. J. 24, 1169-1190 (1975)

67. Fedosov, B.V.: Index theorems. In: Partial Differential Equations, VIII Encyclopaedia Math. Sci., vol. 65. Springer Verlag, Berlin (1996)

68. Feng, B., Sugino, O., Liu, R.Y., Zhang, J., Yukawa, R., Kawamura, M., Iimori, T., Kim, H., Hasegawa, Y., Li, H., Chen, L., Wu, K., Kumigashira, H., Komori, F., Chiang, T.C., Meng, S., Matsuda, I.: Dirac fermions in borophene. Phys. Rev. Lett. 118, 096401 (2017)

69. Fialko, O., Brand, J., Zülicke, U.: Hidden long-range order in a two-dimensional spin-orbit coupled bose gas. Phys. Rev. A 85, 051605(R) (2012)

70. Finkelstein, R., Lelevier, R., Ruderman, M.: Nonlinear spinor fields. Phys. Rev. 83, 326-332 (1951)

71. Fring, A., Jones, H., Znojil, M.: Papers dedicated to the subject of the 6th international workshop on pseudo-Hermitian Hamiltonians in quantum physics (PHHQPVI). J. Phys. A: Math Theor. 41(44) (2008)

72. Galindo, A.: A remarkable invariance of classical Dirac Lagrangians. Lett. Nuovo Cimento 20, 210-212 (1977)

73. Georgiev, V., Ohta, M.: Nonlinear instability of linearly unstable standing waves for nonlinear Schrödinger equations. J. Math. Soc. Japan 64(2), 533-548 (2012)

74. Grillakis, M., Shatah, J., Strauss, W.: Stability theory of solitary waves in the presence of symmetry. I. J. Funct. Anal. 74(1), 160-197 (1987)

75. Gross, D.J., Neveu, A.: Dynamical symmetry breaking in asymptotically free field theories. Phys. Rev. D 10, 3235-3253 (1974) 
76. Gross, L.: The Cauchy problem for the coupled Maxwell and Dirac equations. Comm. Pure Appl. Math. 19, 1-15 (1966)

77. Guo, A., Salamo, G.J., Duchesne, D., Morandotti, R., Volatier-Ravat, M., Aimez, V., Siviloglou, G.A., Christodoulides, D.N.: Observation of PT-symmetry breaking in complex optical potentials. Phys. Rev. Lett. 103, 093902 (2009)

78. Haddad, L.H., Carr, L.D.: The nonlinear Dirac equation in Bose-Einstein condensates: vortex solutions and spectra in a weak harmonic trap. New J. Phys. 17, 113011 (2015)

79. Haddad, L.H., O'Hara, K.M., Carr, L.D.: Nonlinear Dirac equation in Bose-Einstein condensates: preparation and stability of relativistic vortices. Phys. Rev. A 91, 043609 (2015)

80. Hairer, E., Nørsett, S.P., Wanner, G.: Solving Ordinary Differential Equations I, Springer Series in Computational Mathematics, vol. 8. Springer, Berlin (1993)

81. Heisenberg, W.: Quantum theory of fields and elementary particles. Rev. Mod. Phys. 29, 269-278 (1957)

82. Herring, G., Carr, L.D., Carretero-González, R., Kevrekidis, P.G., Frantzeskakis, D.J.: Radially symmetric nonlinear states of harmonically trapped Bose-Einstein condensates. Phys. Rev. A 77, 023625 (2008)

83. Huh, H.: Global solutions to Gross-Neveu equation. Lett. Math. Phys. 103(8), 927-931 (2013)

84. Ivanenko, D.D.: Notes to the theory of interaction via particles. Sov. Phys. JETP 13, 141 (1938)

85. Jensen, A., Kato, T.: Spectral properties of Schrödinger operators and time-decay of the wave functions. Duke Math. J. 46(3), 583-611 (1979)

86. Johansson, M., Kivshar, Y.S.: Discreteness-induced oscillatory instabilities of dark solitons. Phys. Rev. Lett. 82, 85-88 (1999)

87. Jones, C.: Stability of the travelling wave solutions of the Fitzhugh-Nagumo system. Trans. AMS 286(2), 431-469 (1984)

88. Kapitula, T., Sandstede, B.: Edge bifurcations for near integrable systems via Evans function techniques. SIAM J. Math. Anal. 33(5), 1117-1143 (2002)

89. Kartashov, Y.V., Konotop, V.V., Abdullaev, F.K.: Gap solitons in a spin-orbit-coupled boseeinstein condensate. Phys. Rev. Lett. 111, 060402 (2013)

90. Kawakami, T., Mizushima, T., Nitta, M., Machida, K.: Stable skyrmions in SU(2) gauged Bose-Einstein condensates. Phys. Rev. Lett. 109, 015301 (2012)

91. Kestelman, H.: Anticommuting linear transformations. Canad. J. Math. 13, 614-624 (1961)

92. Kevrekidis, P.G., Frantzeskakis, D.J., Carretero-González, R.: The defocusing Nonlinear Schrödinger equation: From dark solitons, to vortices and vortex rings. SIAM, Philadelphia (2015)

93. Kharif, C., Pelinovsky, E., Slunyaev, A.: Rogue Waves in the Ocean. Springer-Verlag, Berlin (2009)

94. Kivshar, Y.S., Agrawal, G.P.: Optical solitons: from fibers to photonic crystals. Academic Press, San Diego (2003)

95. Klaiman, S., Günther, U., Moiseyev, N.: Visualization of branch points in PT-symmetric waveguides. Phys. Rev. Lett. 101, 080402 (2008)

96. LeBlanc, L.J., Beeler, M.C., Jiménez-García, K., Perry, A.R., Sugawa, S., Williams, R.A., Spielman, I.B.: Direct observation of zitterbewegung in a Bose-Einstein condensate. New J. Phys. 15, 073011 (2013)

97. Lee, S.Y., Kuo, T.K., Gavrielides, A.: Exact localized solutions of two-dimensional field theories of massive fermions with Fermi interactions. Phys. Rev. D 12, 2249-2253 (1975)

98. Lee, Y.S., McLean, A.D.: Relativistic effects on $R_{e}$ and $D_{e}$ in $\mathrm{AgH}$ and $\mathrm{AuH}$ from all-electron Dirac-Hartree-Fock calculations. The Journal of Chemical Physics 76(1), 735-736 (1982). DOI 10.1063/1.442680

99. Lieb, E.H.: Existence and uniqueness of the minimizing solution of Choquard's nonlinear equation. Studies in Appl. Math. 57(2), 93-105 (1977)

100. Lin, Y.J., Jiménez-García, K., Spielman, I.B.: Spin-orbit-coupled Bose-Einstein condensates. Nature 471, 83-86 (2011) 
101. Machihara, S., Nakamura, M., Nakanishi, K., Ozawa, T.: Endpoint Strichartz estimates and global solutions for the nonlinear Dirac equation. J. Funct. Anal. 219(1), 1-20 (2005)

102. Machihara, S., Nakanishi, K., Tsugawa, K.: Well-posedness for nonlinear Dirac equations in one dimension. Kyoto J. Math. 50(2), 403-451 (2010)

103. Mak, K.F., Lee, C., Hone, J., Shan, J., Heinz, T.F.: Atomically thin $\mathrm{MoS}_{2}$ : A new direct-gap semiconductor. Phys. Rev. Lett. 105, 136805 (2010)

104. Makris, K.G., El-Ganainy, R., Christodoulides, D.N., Musslimani, Z.H.: Beam dynamics in PT symmetric optical lattices. Phys. Rev. Lett. 100, 103904 (2008)

105. Makris, K.G., El-Ganainy, R., Christodoulides, D.N., Musslimani, Z.H.: PT-symmetric periodic optical potentials. Int. J. Theor. Phys. 50, 1019-1041 (2011)

106. Marini, A., Longhi, S., Biancalana, F.: Optical simulation of neutrino oscillations in binary waveguide arrays. Phys. Rev. Lett. 113, 150,401 (2014)

107. Mathieu, P., Morris, T.F.: Charged spinor solitons. Canad. J. Phys. 64(3), 232-238 (1986)

108. Merkl, M., Jacob, A., Zimmer, F.E., Öhberg, P., Santos, L.: Chiral confinement in quasirelativistic bose-einstein condensates. Phys. Rev. Lett. 104, 073603 (2010)

109. Merle, F.: Existence of stationary states for nonlinear Dirac equations. J. Differential Equations 74, 50-68 (1988)

110. Merle, F.: Construction of solutions with exactly $\mathrm{k}$ blow-up points for the Schrödinger equation with critical nonlinearity. Commun. Math. Phys. 129, 223-240 (1990)

111. Mertens, F.G., Quintero, N.R., Cooper, F., Khare, A., Saxena, A.: Nonlinear dirac equation solitary waves in external fields. Phys. Rev. E 86, 046602 (2012)

112. Ng, W., Parwani, R.: Nonlinear dirac equations. SIGMA 3, 023 (2009)

113. Pauli, W.: Contributions mathématiques à la théorie des matrices de Dirac. Ann. Inst. H. Poincaré 6, 109-136 (1936)

114. Pego, R.L., Weinstein, M.I.: Eigenvalues, and instabilities of solitary waves. Philos. Trans. Roy. Soc. London Ser. A 340(1656), 47-94 (1992)

115. Peleg, O., Bartal, G., Freedman, B., Manela, O., Segev, M., Christodoulides, D.N.: Conical diffraction and gap solitons in honeycomb photonic lattices. Phys. Rev. Lett. 98, 103901 (2007)

116. Pelinovsky, D.: Survey on global existence in the nonlinear Dirac equations in one spatial dimension. In: Harmonic analysis and nonlinear partial differential equations, RIMS Kôkyûroku Bessatsu, B26, pp. 37-50. Res. Inst. Math. Sci. (RIMS), Kyoto (2011)

117. Pelinovsky, D., Shimabukuro, Y.: Transverse instability of line solitons in massive Dirac equations. J. Nonlinear Sci. 26, 365-403 (2016)

118. Pelinovsky, D.E., Shimabukuro, Y.: Orbital stability of Dirac solitons. Lett. Math. Phys. 104, 21-41 (2014)

119. Pelinovsky, D.E., Stefanov, A.: Asymptotic stability of small gap solitons in nonlinear Dirac equations. J. Math. Phys. 53, 073705 (2012)

120. Peng, B., Özdemir, S.K., Lei, F., Monifi, F., Gianfreda, M., Long, G.L., Fan, S., Nori, F., Bender, C.M., Yang, L.: Parity-time-symmetric whispering-gallery microcavities. Nat. Phys. 10, 394-398 (2014)

121. Pethick, C.J., Smith, H.: Bose-Einstein condensation in dilute gases. Cambridge University Press, Cambridge (2002)

122. Pitaevskii, L.P., Stringari, S.: Bose-Einstein condensation. Oxford University Press, Oxford (2003)

123. Press, W.H., Flannery, B.P., Teukolsky, S.A., Vetterling, W.T.: Numerical Recipes: the Art of Scientific Computing, 3rd. edition edn. Cambridge University Press, Cambridge (1986)

124. Qu, C., Hamner, C., Gong, M., Zhang, C., Engels, P.: Observation of zitterbewegung in a spin-orbit-coupled Bose-Einstein condensate. Phys. Rev. A 88, 021064(R) (2013)

125. Quiney, H.M., Glushkov, V.N., Wilson, S.: The Dirac equation in the algebraic approximation. IX. Matrix Dirac-Hartree-Fock calculations for the $\mathrm{HeH}$ and $\mathrm{BeH}$ ground states using distributed gaussian basis sets. International Journal of Quantum Chemistry 99(6), 950-962 (2004)

126. Rañada, A.F., Rañada, M.F., Soler, M., Vázquez, L.: Classical electrodynamics of a nonlinear Dirac field with anomalous magnetic moment. Phys. Rev. D 10(2), 517-525 (1974) 
127. Radić, J., Sedrakyan, T.A., Spielman, I.B., Galitski, V.: Vortices in spin-orbit-coupled BoseEinstein condensates. Phys. Rev. A 84, 063604 (2011)

128. Ramachandhran, B., Opanchuk, B., Liu, X.J., Pu, H., Drummond, P.D., Hu, H.: Halfquantum vortex state in a spin-orbit-coupled Bose-Einstein condensate. Phys. Rev. A 85, 023606 (2012)

129. Reed, M., Simon, B.: Methods of modern mathematical physics. IV. Analysis of operators. Academic Press [Harcourt Brace Jovanovich Publishers], New York (1978)

130. Regensburger, A., Bersch, C., Miri, M.A., Onishchukov, G., Christodoulides, D.N., Peschel, U.: Parity-time synthetic photonic lattices. Nature 488, 167-171 (2012)

131. Rota Nodari, S.: Perturbation method for particle-like solutions of the Einstein-Dirac equations. Ann. Henri Poincaré 10(7), 1377-1393 (2010)

132. Rota Nodari, S.: Perturbation method for particle-like solutions of the Einstein-DiracMaxwell equations. C. R. Math. Acad. Sci. Paris 348(13-14), 791-794 (2010)

133. Ruschhaupt, A., Delgado, F., Muga, J.G.: Physical realization of PT-symmetric potential scattering in a planar slab waveguide. J. Phys. A: Math. Gen. 38, L171-L176 (2005)

134. Rüter, C.E., Makris, K.G., El-Ganainy, R., Christodoulides, D.N., Segev, M., Kip, D.: Observation of parity-time symmetry in optics. Nat. Phys. 6, 192-195 (2010)

135. Sakaguchi, H., Li, B., Malomed, B.A.: Creation of two-dimensional composite solitons in spin-orbit-coupled self-attractive bose-einstein condensates in free space. Phys. Rev. E 89, 032920 (2014)

136. Schindler, J., Li, A., Zheng, M.C., Ellis, F.M., Kottos, T.: Experimental study of active LRC circuits with PT symmetries. Phys. Rev. A 84, 040101 (2011)

137. Schindler, J., Lin, Z., Lee, J.M., Ramezani, H., Ellis, F.M., Kottos, T.: PT-symmetric electronics. J. Phys. A: Math. Theor. 45, 444029 (2012)

138. Selberg, S., Tesfahun, A.: Low regularity well-posedness for some nonlinear Dirac equations in one space dimension. Differential Integral Equations 23(3-4), 265-278 (2010)

139. Shampine, L.F., Hosea, M.E.: Analysis and implementation of TR-BDF2. App. Num. Math. 20, 21-37 (1996)

140. Shao, S., Quintero, N.R., Mertens, F.G., Cooper, F., Khare, A., Saxena, A.: Stability of solitary waves in the nonlinear Dirac equation with arbitrary nonlinearity. Phys. Rev. E 90, 032915 (2014)

141. Sigal, I.M.: Nonlinear wave and Schrödinger equations. I. Instability of periodic and quasiperiodic solutions. Comm. Math. Phys. 153(2), 297-320 (1993)

142. Soffer, A., Weinstein, M.I.: Resonances, radiation damping and instability in Hamiltonian nonlinear wave equations. Invent. Math. 136(1), 9-74 (1999)

143. Soler, M.: Classical, stable, nonlinear spinor field with positive rest energy. Phys. Rev. D 1, 2766-2769 (1970)

144. Soler, M.: Classical Electrodynamics for a Nonlinear Spinor Field: Perturbative and Exact Approaches. Phys. Rev. D 8, 3424-3429 (1973)

145. Strauss, W.A., Vázquez, L.: Stability under dilations of nonlinear spinor fields. Phys. Rev. D 34(2), 641-643 (1986)

146. Stuart, D.: Existence and Newtonian limit of nonlinear bound states in the Einstein-Dirac system. J. Math. Phys. 51(3), 032501 (2010)

147. Sulem, C., Sulem, P.L.: The Nonlinear Schrödinger Equation. Springer-Verlag, New York (1999)

148. Thaller, B.: The Dirac equation. Texts and Monographs in Physics. Springer-Verlag, Berlin (1992)

149. Thirring, W.E.: A soluble relativistic field theory. Ann. Phys. 3, 91-112 (1958)

150. Trefethen, L.N.: Spectral methods in Matlab. SIAM, Philadelphia (2000)

151. Vakhitov, N.G., Kolokolov, A.A.: Stationary solutions of the wave equation in a medium with nonlinearity saturation. Radiophys. Quantum Electron. 16, 783-789 (1973)

152. Vázquez, L.: Localised solutions of a non-linear spinor field. J. Phys. A: Math. Gen. 10, 1361-1368 (1977)

153. Visscher, L., Dyall, K.: Dirac-Fock atomic enectronic structore calculations using different nuclear charge distributions. Atomic Data and Nuclear Data Tables 67(2), 207-224 (1997) 
154. van der Waerden, B..: Group theory and quantum mechanics. Springer-Verlag, New York (1974)

155. Wakano, M.: Intensely localized solutions of the classical Dirac-Maxwell field equations. Prog. Theor. Phys. 35, 1117-1141 (1966)

156. Wehling, T.O., Black-Schaffer, A.M., Balatsky, A.V.: Dirac materials. Adv. Phys. 63, 1-76 (2014)

157. Xu, J., Shao, S., Tang, H.: Numerical methods for nonlinear Dirac equation. J. Comput. Phys. 245, 131-149 (2013)

158. Xu, X.Q., Han, J.H.: Spin-orbit coupled Bose-Einstein condensate under rotation. Phys. Rev. Lett. 107, 200401 (2011)

159. Xu, Y., Zhang, Y., Wu, B.: Bright solitons in spin-orbit-coupled Bose-Einstein condensates. Phys. Rev. A 87, 013614 (2013) 